\title{
Organic acid production in vitro and plant growth promotion in maize under controlled environment by phosphate-solubilizing fluorescent Pseudomonas Pratibha Vyas and Arvind Gulati*
}

\author{
Address: Plant Pathology and Microbiology Laboratory, Institute of Himalayan Bioresource Technology (CSIR), P.O. Box No. 6, Palampur (H.P.)- \\ 176061 , India \\ Email: Pratibha Vyas - vyasp2000@yahoo.co.in; Arvind Gulati* - gal_arvind@yahoo.co.in \\ * Corresponding author
}

Published: 22 August 2009

BMC Microbiology 2009, 9:174 doi:10.1/86/|47|-2/80-9-174
Received: 16 April 2009

Accepted: 22 August 2009

This article is available from: http://www.biomedcentral.com//47/-2/80/9//74

(C) 2009 Vyas and Gulati; licensee BioMed Central Ltd.

This is an Open Access article distributed under the terms of the Creative Commons Attribution License (http://creativecommons.org/licenses/by/2.0), which permits unrestricted use, distribution, and reproduction in any medium, provided the original work is properly cited.

\begin{abstract}
Background: Phosphorus deficiency is a major constraint to crop production due to rapid binding of the applied phosphorus into fixed forms not available to the plants. Microbial solubilization of inorganic phosphates has been attributed mainly to the production of organic acids. Phosphatesolubilizing microorganisms enhance plant growth under conditions of poor phosphorus availability by solubilizing insoluble phosphates in the soil. This paper describes the production of organic acids during inorganic phosphate solubilization and influence on plant growth as a function of phosphate solubilization by fluorescent Pseudomonas.

Results: Nineteen phosphate-solubilizing fluorescent Pseudomonas strains of $P$. fluorescens, $P$. poae, $P$. trivialis, and Pseudomonas spp. produced gluconic acid, oxalic acid, 2-ketogluconic acid, lactic acid, succinic acid, formic acid, citric acid and malic acid in the culture filtrates during the solubilization of tricalcium phosphate, Mussoorie rock phosphate, Udaipur rock phosphate and North Carolina rock phosphate. The strains differed quantitatively and qualitatively in the production of organic acids during solubilization of phosphate substrates. Cluster analysis based on organic acid profiling revealed inter-species and intra-species variation in organic acids produced by Pseudomonas strains. The phosphate-solubilizing bacterial treatments $P$. trivialis BIHB 745 , P. trivialis BIHB 747, Pseudomonas sp. BIHB 756 and $P$. poae BIHB 808 resulted in significantly higher or statistically at par growth and total $\mathrm{N}, \mathrm{P}$ and $\mathrm{K}$ content over single super phosphate treatment in maize. These treatments also significantly affected $\mathrm{pH}$, organic matter, and $\mathrm{N}, \mathrm{P}$, and $\mathrm{K}$ content of the soil.

Conclusion: The results implied that organic acid production by Pseudomonas strains is independent of their genetic relatedness and each strain has its own ability of producing organic acids during the solubilization of inorganic phosphates. Significant difference in plant growth promotion by efficient phosphate-solubilizing Pseudomonas strains point at the need for selecting potential strains in plant growth promotion experiments in conjunction with various phosphate substrates for their targeted application as bioinoculants.
\end{abstract}




\section{Background}

Phosphorus (P) is an essential macronutrient often limiting the plant growth due to its low solubility and fixation in the soil. Improving soil fertility by releasing bound phosphorus by microbial inoculants is an important aspect for increasing crop yield. Phosphorus release from insoluble phosphates reported for several soil microorganisms has been attributed mainly to the production of organic acids and their chelation capacity [1-3]. Direct periplasmic oxidation of glucose to gluconic acid is considered as the metabolic basis of inorganic phosphate solubilization by many Gram-negative bacteria as a competitive strategy to transform the readily available carbon sources into less readily utilizable products by other microorganisms $[1,4]$.

Increased solubilization of fixed soil phosphates and applied phosphates ensuring higher crop yields has been reported on inoculation of phosphate-solubilizing bacteria including Pseudomonas, Bacillus, Rhizobium, Micrococcus, Flavobacterium, Burkholderia, Achromobacter, Erwinia, and Agrobacterium [5,6]. Several Pseudomonas species have been reported among the most efficient phosphate-solublizing bacteria and as important bio-inoculants due to their multiple biofertilizing activities of improving soil nutrient status, secretion of plant growth regulators, and suppression of soil-borne pathogens [5,7-9].

Soils in the cold deserts of Lahaul and Spiti in the Indian trans-Himalayan region latitude $31^{\circ} 44^{\prime} 57^{\prime \prime}$ and $32^{\circ} 59^{\prime}$ $57^{\prime \prime}$ North and $76^{\circ} 46^{\prime} 29^{\prime \prime}$ and $78^{\circ} 41^{\prime} 34^{\prime \prime}$ East are often with low moisture status, high alkalinity, and low organic matter content. The nutritional problems in such soils are often specific in respect of the low phosphorus availability resulting from their high phosphorus-fixing capacity due to high calcium content [10]. The vast potential of microorganisms for improving productivity in the region remains unexploited [11]. Previously we have reported the isolation, selection, and characterization of stress-tolerant and efficient phosphate-solubilizing fluorescent Pseudomonas from the cold deserts of the Himalayas $[8,9]$. The aim of the present study was to explicate organic acid production during solubilization of inorganic phosphates and effect on plant growth as a function of phosphate solubilization by fluorescent Pseudomonas.

\section{Methods}

\section{Bacterial strains}

Nineteen phosphate-solubilizing fluorescent Pseudomonas included in the present studies were isolated from the rhizosphere of Hippophae rhamnoides growing in the cold deserts of Lahaul and Spiti in the trans-Himalayas and characterized based on their phenotypic characters and $16 \mathrm{~S}$ rDNA gene sequencing $[8,9]$. The bacterial strains were maintained at $-70^{\circ} \mathrm{C}$ in nutrient broth supplemented with $20 \%(\mathrm{v} / \mathrm{v})$ glycerol.

\section{Production of organic acids during phosphate solubilization}

The bacterial strains grown in triplicate in $10 \mathrm{ml}$ NBRIP broth supplemented with $0.5 \%$ tricalcium phosphate (TCP), Mussoorie rock phosphate (MRP), Udaipur rock phosphate (URP) and North Carolina rock phosphate (NCRP) at $28^{\circ} \mathrm{C}$ for 5 days at $180 \mathrm{rpm}$ in a refrigerated incubator shaker (Innova Model 4230, New Brunswick Scientific, USA) were centrifuged at $10,000 \mathrm{rpm}$ for 10 min. and passed through $0.22 \mu \mathrm{m}$ nylon filter. Quantitative estimation of P-liberated from inorganic phosphates was done using vanado-molybdate method as described earlier [8]. Detection and quantification of organic acids was done on Waters 996 High Performance Liquid Chromatogram (HPLC) equipped with PDA detector, Waters 717 plus autosampler, Waters 600 controller, Waters $^{\mathrm{TM}}$ pump, Waters inline degasser AF, and Lichrosphere RP-18 column $250 \mathrm{~mm} \times 4.6 \mathrm{~mm}$ and $5 \mu \mathrm{m}$ particle size (Merck, Germany). The mobile phase was $0.1 \%$ ortho-phosphoric acid (Merck, Germany) in the gradient of flow rate as given in Table 1 . Eluates were detected at $\lambda 210 \mathrm{~nm}$ and identified by retention time and co-chromatography by spiking the sample with the authentic organic acids. The organic acids were quantified by reference to the peak areas obtained for the authentic standards for gluconic acid (Sigma-Aldrich, USA), 2-ketogluconic acid (Sigma, USA), and lactic acid, oxalic acid, malic acid, succinic acid, formic acid, citric acid, malonic acid, propionic acid and tartaric acid (Supelco, USA). Each replicate was analyzed in a single run on HPLC for 76 samples for the four phosphate substrates. The values were presented as the mean of three replicates.

\section{Inoculum preparation}

The bacterial strains grown in $20 \mathrm{ml}$ trypticase soya broth (TSB) for $48 \mathrm{~h}$ at $28^{\circ} \mathrm{C}$ were centrifuged at $10,000 \mathrm{rpm}$ for $10 \mathrm{~min}$. and the pellets suspended in $0.85 \% \mathrm{NaCl}\left(\mathrm{OD}_{600}\right.$ $=1.0$ ). The bacterial suspensions were separately mixed with sterilized activated charcoal $(4: 6 \mathrm{v} / \mathrm{w})$ to give a CFU of approximately $10^{7} / \mathrm{g}$ of charcoal-based bacterial inoculants.

\section{Plant growth under controlled environment}

Seeds of Zea mays var. Girija surface sterilized with 20\% sodium hypochlorite for $3 \mathrm{~min}$. and washed thrice with sterile distilled water were germinated at $25^{\circ} \mathrm{C}$ in moist sterile vermiculite. Uniformly germinated seeds were coated with the water slurry of charcoal-based microbial

Table I: HPLC elution-profile program.

\begin{tabular}{lc}
\hline Time $(\mathrm{min})$ & Flow rate $(\mathrm{ml} / \mathrm{min})$ \\
\hline $0-8$ & 0.4 \\
$8-14$ & 0.5 \\
$14-25$ & 1.2 \\
\hline
\end{tabular}


inoculants (approx. $5 \times 10^{5} \mathrm{CFU} /$ seed) and two seeds per pot sown in $15 \mathrm{~cm}$ diameter pots filled with $2 \mathrm{~kg}$ non-sterilized sandy-loam soil. The soil used had pH 6.96, organic matter $3.1 \%$, available N $0.03 \%$, available P $0.0011 \%$, available $\mathrm{K} 0.013 \%$ and available Ca $0.028 \%$. The germinated seeds treated with the water slurry of sterilized activated charcoal without inoculum were used for the control treatments. $\mathrm{N}$ and $\mathrm{K}$ were applied in the form of ammonium sulfate@ $240 \mathrm{~kg} \mathrm{~N} / \mathrm{ha}$, and muriate of potash @ $80 \mathrm{~kg} \mathrm{~K} / \mathrm{ha}$, respectively. P was applied @ $120 \mathrm{~kg} \mathrm{P} / \mathrm{ha}$ either as single super phosphate (SSP) or tricalcium phosphate (TCP) according to the various treatments. The phosphate-solubilizing bacterial (PSB) treatments included one $P$. fluorescens strain, three $P$. poae strains, ten $P$. trivialis strains, and five Pseudomonas spp. strains in combined application with NPK with TCP as the phosphate source. TCP was chosen as phosphate substrate since P-deficiency in soils of the cold deserts of Lahaul and Spiti is attributed mainly to the presence of insoluble diand tricalcium phosphates. The influence of PSB treatments on plant growth and soil properties was evaluated in comparison to the uninoculated control treatments with or without TCP and SSP. The pots were placed in a complete randomized block design with four replications under $550 \mu \mathrm{M}$ photon $\mathrm{m}^{-2} \mathrm{~s}^{-1}$ mixed incandescent and fluorescent illumination, $16 / 8 \mathrm{~h}$ light/dark cycle and 50$60 \% \mathrm{RH}$ at $25 \pm 2{ }^{\circ} \mathrm{C}$ in an Environment Control Chamber. The plants were removed carefully under a gentle flow of tap water after 90 days of sowing. Data on root length, plant height (aerial parts), root dry weight and shoot dry weight were recorded. The samples were oven-dried at $70^{\circ} \mathrm{C}$ for 3 days to a constant weight for determining the dry weight.

\section{Chemical analyses}

The soil samples were air dried and sieved for determining $\mathrm{pH}$, available $\mathrm{N}, \mathrm{P}, \mathrm{K}, \mathrm{Ca}$ and organic matter content. The plant samples were oven-dried and powdered for estimation of total N, P and K. Organic matter was determined by the modified Walkley and Black method [12]. Estimation of total $\mathrm{N}$ was done by modified Kjeldahl's method, total $P$ by vanado-molybdate yellow colour method, total and available $\mathrm{K}$ by flame photometric method, and available Ca in ammonium-acetate extracts [13]. Estimation of available $\mathrm{P}$ was estimated by sodium biocarbonate method [14] and available $\mathrm{N}$ by alkaline permanganate method [15].

\section{Experimental design and data analyses}

Randomized block design with two factor factorial arrangement was adopted for conducting the experiments. The data were subjected to one-way analysis of variance (ANOVA) and the mean of treatments compared by Duncan's Multiple Range Test at $\mathrm{p} \leq 0.01$ using SPSS Software version 7.5. Cluster analysis based on the organic acid profiles was performed using STATISTICA data analysis software system version 7 (StatSoft ${ }^{\circledast}$ Inc. Tulsa, USA, 2004).

\section{Results \\ Production of organic acids}

HPLC analysis of the culture filtrates was done to identify and quantity the organic acids produced during the solubilization of TCP, MRP, URP and NCRP by Pseudomonas fluorescens strain, three Pseudomonas poae strains, ten Pseudomonas trivialis strains, and five Pseudomonas spp. strains (Fig. 1). During TCP solubilization all strains showed the production of gluconic and 2-ketogluconic acids (Table 2). Apart from one Pseudomonas sp. strain no other strain showed oxalic acid production. All strains exhibited the production of malic acid excepting one Pseudomonas sp. strain and succinic acid excluding one Pseudomonas sp. strain. The production of lactic acid was restricted to one strain of both P. trivialis and Pseudomonas sp., formic acid to six P. trivialis, P. fluorescens and two Pseudomonas spp. strains, and citric acid to three $P$. trivialis strains and one strain each of $P$. poae and Pseudomonas sp., and P. fluorescens strain.

During URP solubilization the production of oxalic and gluconic acid was detected for all the strains (Table 3). The production of other organic acids was restricted to some strains: 2-ketogluconic acid to three Pseudomonas spp. strains and one strain each of $P$. trivialis, $P$. poae and $P$. fluorescens; lactic acid to five P. trivialis, $P$. fluorescens and two Pseudomonas spp. strains; succinic acid to one strain each of $P$. trivialis, $P$. fluorescens and Pseudomonas sp.; formic acid to two P. trivialis strains; and malic acid to four $P$. trivialis, two P. poae and four Pseudomonas spp. strains. None of the strains showed citric acid production during URP solubilization.

During MRP solubilization the production of oxalic and gluconic acid was also detected for all the strains (Table 4 ). The production of 2-ketogluconic acid was shown by one Pseudomonas poae, P. fluorescens and four Pseudomonas spp. strains, lactic acid by five $P$. trivialis, one $P$. poae and three Pseudomonas spp. strains, succinic acid by three Pseudomonas spp. strains, formic acid by three $P$. trivialis and three Pseudomonas spp. strains, formic acid by $P$. fluorescens and three $P$. trivialis strains, malic acid by two $P$. trivialis, one P. poae, P. fluorescens and four Pseudomonas spp. strains, and citric acid by one Pseudomonas sp. strain.

In NCRP solubilization the production of oxalic acid and gluconic acid was detected for all the strains (Table 5). The production of other organic acids was limited to some strains: 2-ketogluconic acid to five $P$. trivialis, two $P$. poae, P. fluorescens and three Pseudomonas spp. strains; lactic acid to three $P$. trivialis and four Pseudomonas spp. strains; 

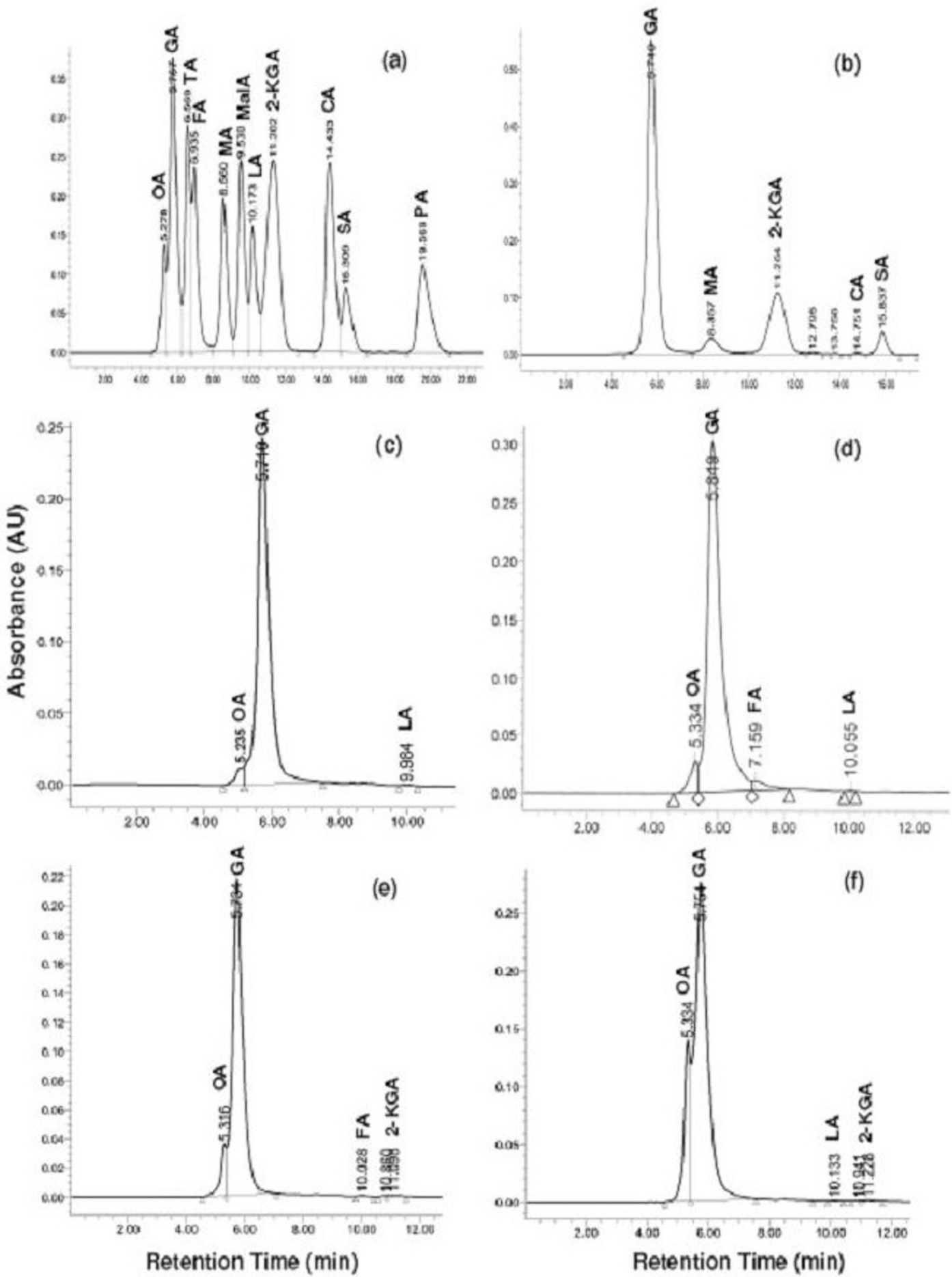

Figure I

HPLC chromatograms of authentic organic acids (a) and culture supernatant of Pseudomonas trivialis strain BIHB 747 grown for 5 days at $28^{\circ} \mathrm{C}$ in NBRIP broth with tricalcium phosphate (b), Udaipur rock phosphate (c), Mussoorie rock phosphate (d), North Carolina rock phosphate (e), and North Carolina rock phosphate spiked with OA (f). OA = oxalic acid, $\mathrm{GA}=$ gluconic acid, TA = tartaric acid, $\mathrm{FA}=$ formic acid, $\mathrm{MA}=$ malic acid, $\mathrm{MalA}=$ malonic acid, $\mathrm{LA}=$ lactic acid, 2-KGA = 2-ketogluconic acid, $\mathrm{SA}=$ succinic acid, $\mathrm{CA}=$ citric acid and $\mathrm{PA}=$ propionic acid. 
Table 2: Organic acid production by fluorescent Pseudomonas during tricalcium phosphate solubilization.

\begin{tabular}{|c|c|c|c|c|c|c|c|c|c|c|c|}
\hline \multirow[b]{2}{*}{ Strain } & \multirow[b]{2}{*}{$\begin{array}{l}\text { P-liberated } \\
(\mu \mathrm{g} / \mathrm{ml})\end{array}$} & \multirow[b]{2}{*}{ Final $\mathrm{pH}$} & \multicolumn{8}{|c|}{ Organic acid $(\mu g / \mathrm{ml})$} & \multirow[b]{2}{*}{$\begin{array}{l}\text { Total organic } \\
\text { acids }(\mu \mathrm{g} / \mathrm{ml})\end{array}$} \\
\hline & & & Oxalic & Gluconic & 2-KGA & Lactic & Succinic & Formic & Citric & Malic & \\
\hline \multicolumn{12}{|l|}{ P. trivialis } \\
\hline BIHB 728 & $771.3 \pm 1.2$ & 3.63 & ND & $18350.0 \pm 5.8$ & $257.0 \pm 4.9$ & $49.3 \pm 1.8$ & $987.7 \pm 3.0$ & ND & $30.5 \pm 2.8$ & $2051.8 \pm 5.2$ & 21726.3 \\
\hline BIHB 736 & $778.7 \pm 2.4$ & 3.90 & ND & $18035.3 \pm 9.0$ & $177.0 \pm 2.6$ & ND & $583.7 \pm 4.1$ & $96.0 \pm 2.3$ & ND & $1042.0 \pm 3.8$ & 19934.0 \\
\hline BIHB 745 & $827.4 \pm 1.8$ & 3.65 & ND & $18054.3 \pm 8.1$ & $210.0 \pm 2.9$ & ND & $2249.0 \pm 4.4$ & ND & $65.2 \pm 2.6$ & $1654.5 \pm 3.8$ & 22233.0 \\
\hline BIHB 747 & $743.0 \pm 1.7$ & 3.52 & ND & $18216.7 \pm 3.5$ & $330.7 \pm 2.9$ & ND & $1307.7 \pm 4.6$ & ND & $25.5 \pm 2.1$ & $667.0 \pm 3.2$ & 20547.6 \\
\hline BIHB 749 & $801.0 \pm 2.1$ & 3.42 & ND & $17745.3 \pm 7.2$ & $193.7 \pm 3.3$ & ND & $797.6 \pm 1.9$ & $117.5 \pm 2.0$ & ND & $1236.0 \pm 6.2$ & 20090.1 \\
\hline BIHB 750 & $774.3 \pm 1.9$ & 3.82 & ND & $18624.0 \pm 4.6$ & $172.3 \pm 3.7$ & ND & $509.9 \pm 2.7$ & $93.5 \pm 1.7$ & ND & $2012.3 \pm 3.9$ & 21412.0 \\
\hline BIHB 757 & $775.3 \pm 2.3$ & 3.92 & ND & $17819.0 \pm 6.7$ & $224.5 \pm 2.6$ & ND & $772.3 \pm 3.4$ & $132.0 \pm 3.5$ & ND & $911.0 \pm 6.1$ & 19858.8 \\
\hline BIHB 759 & $751.3 \pm 3.7$ & 3.72 & ND & $18336.3 \pm 4.5$ & $179.0 \pm 2.9$ & ND & $779.0 \pm 5.0$ & $116.0 \pm 3.2$ & ND & $2551.0 \pm 4.9$ & 21961.3 \\
\hline BIHB 763 & $718.0 \pm 1.5$ & 4.00 & ND & $|790| .3 \pm 5.9$ & $173.7 \pm 2.6$ & ND & $659.7 \pm 4.1$ & $106.0 \pm 5.0$ & ND & $2656.0 \pm 2.7$ & 21496.7 \\
\hline BIHB 769 & $806.4 \pm 2.3$ & 3.70 & ND & $19340.0 \pm 5.8$ & $154.0 \pm 2.5$ & ND & $207.7 \pm 3.8$ & ND & ND & $1965.0 \pm 5.1$ & 21666.7 \\
\hline \multicolumn{12}{|l|}{ P. poae } \\
\hline BIHB 730 & $768.3 \pm 1.8$ & 3.40 & ND & $17464.7 \pm 5.5$ & $251.0 \pm 3.1$ & ND & $1172.7 \pm 5.9$ & ND & ND & $|7| 8.8 \pm 3.4$ & 20607.2 \\
\hline BIHB 752 & $805.0 \pm 1.7$ & 3.50 & ND & $18800.7 \pm 6.4$ & $217.0 \pm 4.2$ & ND & $321.3 \pm 4.1$ & ND & ND & $3128.0 \pm 4.5$ & 22467.0 \\
\hline BIHB 808 & $821.4 \pm 1.7$ & 3.58 & ND & $18840.3 \pm 7.3$ & $176.3 \pm 2.3$ & ND & $475.7 \pm 6.6$ & ND & $44.3 \pm 2.9$ & $75.0 \pm 3.6$ & 19611.6 \\
\hline \multicolumn{12}{|c|}{ P. fluorescens } \\
\hline BIHB 740 & $768.3 \pm 2.6$ & 3.97 & ND & $17038.7 \pm 3.8$ & $175.3 \pm 4.4$ & ND & $163.3 \pm 3.5$ & $129.0 \pm 3.8$ & $46.0 \pm 3.2$ & $3178.0 \pm 3.8$ & 20730.3 \\
\hline \multicolumn{12}{|c|}{ Pseudomonas spp. } \\
\hline BIHB 75I & $318.7 \pm 2.0$ & 4.20 & $7.7 \pm 0.6$ & $216.7 \pm 3.5$ & $532.3 \pm 4.3$ & ND & ND & $23.8 \pm 1.7$ & ND & $1181.0 \pm 5.9$ & 1961.5 \\
\hline BIHB 756 & $802.3 \pm 2.1$ & 3.53 & ND & $17937.3 \pm 6.2$ & $378.0 \pm 3.6$ & ND & $209.4 \pm 3.2$ & ND & ND & $4215.0 \pm 3.2$ & 22739.7 \\
\hline BIHB 804 & $805.1 \pm 2.2$ & 3.55 & ND & $\mid 7929.7 \pm 4.1$ & $122.7 \pm 2.4$ & $53.7 \pm 1.8$ & $96.0 \pm 2.5$ & ND & ND & $1520.0 \pm 3.8$ & 19722.1 \\
\hline BIHB 8I I & $717.3 \pm 1.9$ & 3.98 & ND & $14427.3 \pm 2.3$ & $14.3 \pm 0.4$ & ND & $195.3 \pm 4.3$ & ND & $28.5 \pm 1.8$ & ND & 14665.4 \\
\hline BIHB 813 & $631.7 \pm 2.5$ & 3.93 & ND & $18057.7 \pm 5.4$ & $175.3 \pm 5.9$ & ND & $536.3 \pm 4.5$ & $1 \mathrm{I} 4.4 \pm 4.4$ & ND & $913.7 \pm 3.7$ & 19797.4 \\
\hline Total orga & $\mathrm{s}(\mu \mathrm{g} / \mathrm{ml})$ & & 7.7 & 323135.3 & $4 \mid 14.1$ & 103.0 & 12024.3 & 928.2 & 240.0 & 32676.1 & 373228.7 \\
\hline
\end{tabular}

Values are the mean of three replicates \pm standard error of the mean; ND = not detected; 2-KGA = 2-ketogluconic acid. 
Table 3: Organic acid production by fluorescent Pseudomonas during Udaipur rock phosphate solubilization.

\begin{tabular}{|c|c|c|c|c|c|c|c|c|c|c|c|}
\hline \multirow[b]{2}{*}{ Strain } & \multirow[b]{2}{*}{$\begin{array}{l}\text { P-liberated } \\
(\mu \mathrm{g} / \mathrm{ml})\end{array}$} & \multirow[b]{2}{*}{ Final $\mathrm{pH}$} & \multicolumn{8}{|c|}{ Organic acid $(\mu \mathrm{g} / \mathrm{ml})$} & \multirow[b]{2}{*}{$\begin{array}{l}\text { Total organic } \\
\text { acids }(\mu g / \mathrm{ml})\end{array}$} \\
\hline & & & Oxalic & Gluconic & 2-KGA & Lactic & Succinic & Formic & Citric & Malic & \\
\hline \multicolumn{12}{|l|}{ P. trivialis } \\
\hline BIHB 728 & $8.7 \pm 0.04$ & 3.78 & $14.3 \pm 1.5$ & $6676.7 \pm 6.0$ & ND & $52.8 \pm 1.3$ & ND & ND & ND & ND & 6743.8 \\
\hline BIHB 736 & $5.6 \pm 0.10$ & 3.79 & $10.6 \pm 1.5$ & $7116.0 \pm 5.9$ & ND & ND & ND & ND & ND & ND & 7126.6 \\
\hline BIHB 745 & $8.3 \pm 0.30$ & 3.78 & $11.1 \pm 0.9$ & $8190.0 \pm 5.8$ & ND & ND & ND & $35.1 \pm 3.1$ & ND & $53.4 \pm 3.7$ & 8289.6 \\
\hline BIHB 747 & $4.4 \pm 0.01$ & 3.71 & $10.3 \pm 1.1$ & $6962.3 \pm 5.0$ & ND & $41.3 \pm 2.0$ & ND & ND & ND & ND & 7013.9 \\
\hline BIHB 749 & $5.3 \pm 0.01$ & 3.60 & $11.4 \pm 0.7$ & $7921.7 \pm 6.9$ & ND & $41.3 \pm 3.5$ & ND & ND & ND & ND & 7974.4 \\
\hline BIHB 750 & $6.1 \pm 0.02$ & 3.87 & $9.4 \pm 0.8$ & $7496.7 \pm 6.0$ & ND & ND & ND & ND & ND & $34.5 \pm 2.5$ & 7540.6 \\
\hline BIHB 757 & $7.1 \pm 0.04$ & 3.72 & $8.7 \pm 1.2$ & $5459.0 \pm 3.1$ & ND & ND & ND & ND & ND & ND & 5467.7 \\
\hline BIHB 759 & $14.0 \pm 0.90$ & 3.62 & $9.5 \pm 1.0$ & $6850.0 \pm 6.4$ & ND & ND & ND & ND & ND & ND & 6859.5 \\
\hline BIHB 763 & $9.3 \pm 0.04$ & 3.78 & $26.6 \pm 0.7$ & $10903.0 \pm 3.6$ & ND & $42.8 \pm 1.0$ & ND & $93.6 \pm 2.0$ & ND & $103.6 \pm 3.3$ & 11169.6 \\
\hline BIHB 769 & $7.6 \pm 0.50$ & 3.70 & $12.4 \pm 1.5$ & $2964.0 \pm 3.1$ & $20.5 \pm 2.3$ & $92.3 \pm 1.8$ & $56.1 \pm 4.6$ & ND & ND & $383.0 \pm 3.1$ & 3528.3 \\
\hline \multicolumn{12}{|l|}{ P. poae } \\
\hline BIHB 730 & $5.0 \pm 0.09$ & 3.70 & $25.7 \pm 1.4$ & $5055.3 \pm 5.0$ & $16.4 \pm 1.2$ & ND & ND & ND & ND & ND & 5097.4 \\
\hline BIHB 752 & $7.7 \pm 0.10$ & 3.90 & $8.0 \pm 0.8$ & $7119.0 \pm 3.8$ & ND & ND & ND & ND & ND & $35.5 \pm 3.4$ & 7162.5 \\
\hline BIHB 808 & $7.6 \pm 0.05$ & 3.83 & $9.5 \pm 1.3$ & $7616.3 \pm 3.5$ & ND & ND & ND & ND & ND & $36.3 \pm 3.3$ & 7662.1 \\
\hline \multicolumn{12}{|c|}{ P. fluorescens } \\
\hline BIHB 740 & $3.8 \pm 0.05$ & 4.00 & $12.7 \pm 1.0$ & $1117.7 \pm 5.4$ & $67.0 \pm 2.6$ & $164.0 \pm 2.6$ & $102.3 \pm 1.5$ & ND & ND & ND & | 463.7 \\
\hline \multicolumn{12}{|c|}{ Pseudomonas spp. } \\
\hline BIHB 75I & $1.4 \pm 0.03$ & 4.20 & $13.9 \pm 0.8$ & $631.7 \pm 4.4$ & $255.0 \pm 5.1$ & ND & ND & ND & ND & $4350.0 \pm 2.5$ & 5250.6 \\
\hline BIHB 756 & $9.4 \pm 0.05$ & 3.75 & $11.9 \pm 0.8$ & $5061.7 \pm 9.4$ & $51.7 \pm 2.5$ & ND & ND & ND & ND & $57.7 \pm 2.7$ & 5183.0 \\
\hline BIHB 804 & $3.8 \pm 0.40$ & 4.03 & $12.5 \pm 0.9$ & $5839.3 \pm 7.8$ & ND & $43.2 \pm 2.0$ & ND & ND & ND & $41.8 \pm 2.5$ & 5936.8 \\
\hline BIHB 8II & $6.1 \pm 0.05$ & 4.11 & $17.1 \pm 1.2$ & $44 \mid 2.3 \pm 5.2$ & $138.8 \pm 0.9$ & $121.3 \pm 1.5$ & $108.0 \pm 3.1$ & ND & ND & $658.1 \pm 2.3$ & 5455.6 \\
\hline $\mathrm{BIHB} 813$ & $5.2 \pm 0.30$ & 4.32 & $12.0 \pm 1.5$ & $5971.7 \pm 5.2$ & ND & ND & ND & ND & ND & ND & 5983.7 \\
\hline Total orgar & ml) & & 235.6 & 97392.7 & 549.4 & 599 & 266.4 & 128.7 & 0 & 5753.9 & 104925.7 \\
\hline
\end{tabular}

Values are the mean of three replicates \pm standard error of the mean; ND = not detected; 2-KGA $=2$-ketogluconic acid. 
Table 4: Organic acid production by fluorescent Pseudomonas during Mussoorie rock phosphate solubilization.

\begin{tabular}{|c|c|c|c|c|c|c|c|c|c|c|c|}
\hline \multirow[b]{2}{*}{ Strain } & \multirow[b]{2}{*}{$\begin{array}{c}\text { P-liberated } \\
(\mu \mathrm{g} / \mathrm{ml})\end{array}$} & \multirow[b]{2}{*}{ Final $\mathrm{pH}$} & \multicolumn{8}{|c|}{ Organic acid $(\mu \mathrm{g} / \mathrm{ml})$} & \multirow[b]{2}{*}{$\begin{array}{l}\text { Total organic } \\
\text { acids }(\mu \mathrm{g} / \mathrm{ml})\end{array}$} \\
\hline & & & Oxalic & Gluconic & 2-KGA & Lactic & Succinic & Formic & Citric & Malic & \\
\hline \multicolumn{12}{|l|}{ P. trivialis } \\
\hline BIHB 728 & $11.0 \pm 0.3$ & 3.52 & $15.1 \pm 1.4$ & $8443.3 \pm 6.0$ & ND & $44.9 \pm 1.7$ & ND & ND & ND & ND & 8503.3 \\
\hline BIHB 736 & $13.1 \pm 0.1$ & 3.52 & $15.6 \pm 1.4$ & $93 \mid 4.3 \pm 7.4$ & ND & ND & ND & ND & ND & ND & 9329.9 \\
\hline BIHB 745 & $5.8 \pm 0.3$ & 3.63 & $14.8 \pm 1.4$ & $9394.0 \pm 8.3$ & ND & ND & ND & $84.0 \pm 3.1$ & ND & $930.0 \pm 4.2$ & 10422.8 \\
\hline BIHB 747 & $12.0 \pm 0.2$ & 3.49 & $16.3 \pm 0.7$ & $10016.7 \pm 4.4$ & ND & $36.8 \pm 2.0$ & ND & $70.4 \pm 2.7$ & ND & ND & 10140.2 \\
\hline BIHB 749 & $8.0 \pm 0.04$ & 3.59 & $15.8 \pm 0.7$ & $12027.0 \pm 5.7$ & ND & ND & ND & ND & ND & ND & I2042.8 \\
\hline BIHB 750 & $4.8 \pm 0.4$ & 3.67 & $11.7 \pm 0.9$ & $8460.0 \pm 5.8$ & ND & ND & ND & ND & ND & $32.3 \pm 2.1$ & 8504.0 \\
\hline BIHB 757 & $9.0 \pm 0.04$ & 3.63 & $10.6 \pm 1.0$ & $9460.0 \pm 5.5$ & ND & $39.4 \pm 2.8$ & ND & ND & ND & ND & 9510.0 \\
\hline BIHB 759 & $11.0 \pm 0.2$ & 3.52 & $16.7 \pm 1.3$ & $13854.0 \pm 4.9$ & ND & $39.7 \pm 1.3$ & ND & ND & ND & ND & 13910.4 \\
\hline BIHB 763 & $12.9 \pm 0.02$ & 3.50 & $18.2 \pm 0.5$ & $13444.0 \pm 5.5$ & ND & ND & ND & $87.7 \pm 3.0$ & ND & ND & 13549.9 \\
\hline BIHB 769 & $6.1 \pm 0.4$ & 3.65 & $16.4 \pm 0.7$ & $11633.7 \pm 5.4$ & ND & $40.5 \pm 2.6$ & ND & ND & ND & ND & 11690.6 \\
\hline \multicolumn{12}{|l|}{ P. poae } \\
\hline BIHB 730 & $4.0 \pm 0.06$ & 4.62 & $12.5 \pm 1.3$ & $7871.0 \pm 8.5$ & $19.9 \pm 1.4$ & $37.8 \pm 2.1$ & ND & ND & ND & ND & 7941.2 \\
\hline BIHB 752 & $6.0 \pm 0.03$ & 3.62 & $19.6 \pm 2.1$ & $15727.0 \pm 5.9$ & ND & ND & ND & ND & ND & $293.0 \pm 4.7$ & 16039.6 \\
\hline BIHB 808 & $8.6 \pm 0.6$ & 3.53 & $15.3 \pm 1.2$ & $13749.7 \pm 3.4$ & ND & ND & ND & ND & ND & ND & 13765.0 \\
\hline \multicolumn{12}{|c|}{ P. fluorescens } \\
\hline BIHB 740 & $3.0 \pm 0.1$ & 5.90 & $14.3 \pm 0.9$ & $8051.0 \pm 6.1$ & $468.0 \pm 3.1$ & ND & ND & $114.4 \pm 4.9$ & ND & $183.2 \pm 4.9$ & 8830.9 \\
\hline \multicolumn{12}{|c|}{ Pseudomonas spp. } \\
\hline BIHB 75I & $2.4 \pm 0.1$ & 3.89 & $11.7 \pm 0.4$ & $7076.3 \pm 4.6$ & $126.3 \pm 7.2$ & ND & ND & ND & ND & $2802.0 \pm 4.7$ & 10016.3 \\
\hline BIHB 756 & $12.7 \pm 0.4$ & 3.53 & $14.7 \pm 1.2$ & $9120.0 \pm 6.4$ & $153.0 \pm 3.1$ & ND & $142.0 \pm 3.5$ & ND & ND & $264.0 \pm 4.6$ & 9693.7 \\
\hline BIHB 804 & $8.1 \pm 0.3$ & 3.55 & $39.3 \pm 1.5$ & $8997.0 \pm 7.2$ & $18.4 \pm 0.9$ & $39.6 \pm 1.1$ & ND & ND & ND & $34.1 \pm 2.9$ & 9128.4 \\
\hline BIHB 8II & $2.9 \pm 0.03$ & 4.00 & $42.0 \pm 1.7$ & $10007.0 \pm 3.8$ & $234.3 \pm 2.0$ & $50.8 \pm 2.3$ & $349.7 \pm 2.7$ & ND & $22.3 \pm 2.2$ & $36.1 \pm 2.8$ & 10742.2 \\
\hline BIHB 813 & $2.2 \pm 0.4$ & 4.05 & $14.2 \pm 0.7$ & $10396.0 \pm 5.6$ & ND & $40.5 \pm 2.0$ & $136.0 \pm 2.1$ & ND & ND & ND & 10586.7 \\
\hline Total orga & ds $(\mu \mathrm{g} / \mathrm{ml})$ & & 334.8 & 197042.0 & 1019.9 & 370.0 & 627.7 & 356.5 & 22.3 & 4574.7 & 204347.9 \\
\hline
\end{tabular}

Values are the mean of three replicates \pm standard error of the mean; ND $=$ Not detected; $2-K G A=2$-ketogluconic acid. 
Table 5: Organic acid production by fluorescent Pseudomonas during North Carolina rock phosphate solubilization.

\begin{tabular}{|c|c|c|c|c|c|c|c|c|c|c|c|}
\hline \multirow[b]{2}{*}{ Strain } & \multirow[b]{2}{*}{$\begin{array}{l}\text { P-liberated } \\
(\mu \mathrm{g} / \mathrm{ml})\end{array}$} & \multirow[b]{2}{*}{ Final $\mathrm{pH}$} & \multicolumn{8}{|c|}{ Organic acid $(\mu \mathrm{g} / \mathrm{ml})$} & \multirow[b]{2}{*}{$\begin{array}{l}\text { Total organic } \\
\text { acids }(\mu \mathrm{g} / \mathrm{ml})\end{array}$} \\
\hline & & & Oxalic & Gluconic & 2-KGA & Lactic & Succinic & Formic & Citric & Malic & \\
\hline \multicolumn{12}{|l|}{ P. trivialis } \\
\hline BIHB 728 & $191.3 \pm 1.0$ & 3.70 & $14.7 \pm 0.6$ & $3810.0 \pm 7.6$ & $10.2 \pm 1.0$ & ND & ND & ND & ND & ND & 3834.9 \\
\hline BIHB 736 & $172.0 \pm 0.3$ & 3.72 & $9.1 \pm 1.3$ & $4672.3 \pm 6.4$ & ND & $42.7 \pm 1.2$ & ND & ND & ND & ND & 4724.1 \\
\hline BIHB 745 & $168.2 \pm 0.4$ & 3.73 & $10.8 \pm 0.5$ & $3880.7 \pm 5.2$ & $10.1 \pm 0.8$ & ND & ND & ND & ND & ND & 3901.6 \\
\hline BIHB 747 & $173.0 \pm 0.4$ & 3.81 & $16.6 \pm 1.0$ & $6035.0 \pm 4.2$ & $11.0 \pm 1.8$ & $40.3 \pm 2.9$ & ND & ND & ND & ND & 6102.9 \\
\hline BIHB 749 & $177.3 \pm 0.6$ & 3.73 & $17.1 \pm 0.9$ & $4587.0 \pm 4.7$ & ND & $42.7 \pm 2.2$ & ND & ND & ND & $113.2 \pm 2.7$ & 4760.0 \\
\hline BIHB 750 & $145.7 \pm 1.2$ & 3.88 & $10.3 \pm 0.6$ & $4395.3 \pm 7.7$ & ND & ND & ND & ND & ND & ND & 4405.6 \\
\hline BIHB 757 & $175.0 \pm 0.3$ & 3.92 & $13.6 \pm 2.3$ & $4649.0 \pm 5.5$ & $13.3 \pm 1.1$ & ND & ND & ND & ND & ND & 4675.9 \\
\hline BIHB 759 & $178.0 \pm 0.6$ & 3.81 & $11.0 \pm 1.4$ & $5331.0 \pm 6.1$ & ND & ND & ND & ND & ND & ND & 5342.0 \\
\hline BIHB 763 & $161.2 \pm 0.2$ & 3.80 & $11.5 \pm 1.3$ & $4362.0 \pm 4.6$ & $10.8 \pm 1.0$ & ND & ND & ND & ND & ND & 4384.3 \\
\hline BIHB 769 & $224.0 \pm 0.7$ & 3.55 & $10.8 \pm 0.8$ & $4448.0 \pm 5.3$ & ND & ND & ND & ND & ND & ND & 4458.8 \\
\hline \multicolumn{12}{|l|}{ P. poae } \\
\hline BIHB 730 & $163.8 \pm 1.1$ & 3.90 & $10.1 \pm 1.2$ & $3770.0 \pm 6.4$ & ND & ND & ND & ND & ND & ND & 3780.1 \\
\hline BIHB 752 & $204.3 \pm 0.7$ & 3.72 & $12.7 \pm 1.5$ & $4947.0 \pm 6.0$ & $10.3 \pm 1.0$ & ND & ND & ND & $26.1 \pm 2.0$ & ND & 4996.1 \\
\hline BIHB 808 & $193.4 \pm 0.7$ & 3.65 & $11.5 \pm 1.2$ & $4420.3 \pm 2.9$ & $10.9 \pm 0.8$ & ND & $45.1 \pm 4.3$ & ND & ND & ND & 4442.7 \\
\hline \multicolumn{12}{|c|}{ P. fluorescens } \\
\hline BIHB 740 & $236.8 \pm 0.6$ & 3.48 & $9.8 \pm 1.1$ & $4762.7 \pm 4.3$ & $31.3 \pm 2.0$ & ND & $46.7 \pm 3.2$ & $59.3 \pm 3.5$ & ND & $104.8 \pm 3.0$ & 5014.6 \\
\hline \multicolumn{12}{|c|}{ Pseudomonas spp. } \\
\hline BIHB 75I & $123.3 \pm 1.4$ & 3.89 & $9.1 \pm 1.1$ & $3241.0 \pm 2.6$ & $22.3 \pm 1.9$ & ND & ND & ND & ND & $415.0 \pm 4.0$ & 3687.4 \\
\hline BIHB 756 & $164.2 \pm 0.8$ & 3.82 & $11.3 \pm 0.6$ & $4975.0 \pm 7.5$ & ND & $41.7 \pm 1.4$ & ND & ND & $29.5 \pm 2.2$ & ND & 5057.5 \\
\hline BIHB 804 & $161.5 \pm 1.0$ & 3.78 & $15.7 \pm 1.2$ & $4542.0 \pm 5.3$ & $10.5 \pm 1.0$ & $39.3 \pm 2.0$ & ND & ND & ND & $33.0 \pm 1.2$ & 4640.5 \\
\hline BIHB 8I I & $173.0 \pm 1.1$ & 3.92 & $15.5 \pm 0.8$ & $2549.0 \pm 5.9$ & $32.7 \pm 0.9$ & $54.3 \pm 2.0$ & $75.1 \pm 4.6$ & ND & ND & $265.0 \pm 3.6$ & 2991.6 \\
\hline BIHB 813 & $92.7 \pm 1.2$ & 4.07 & $8.9 \pm 1.2$ & $4633.3 \pm 5.5$ & ND & $38.8 \pm 2.0$ & ND & ND & ND & ND & 4681.0 \\
\hline Total orga & $\mathrm{ds}(\mu \mathrm{g} / \mathrm{ml})$ & & 230.1 & 84010.6 & 173.4 & 299.8 & 121.8 & 59.3 & 55.6 & 931 & 85881.6 \\
\hline
\end{tabular}

Values are the mean of three replicates \pm standard error of the mean; ND $=$ Not detected; 2-KGA $=2$-ketogluconic acid. 

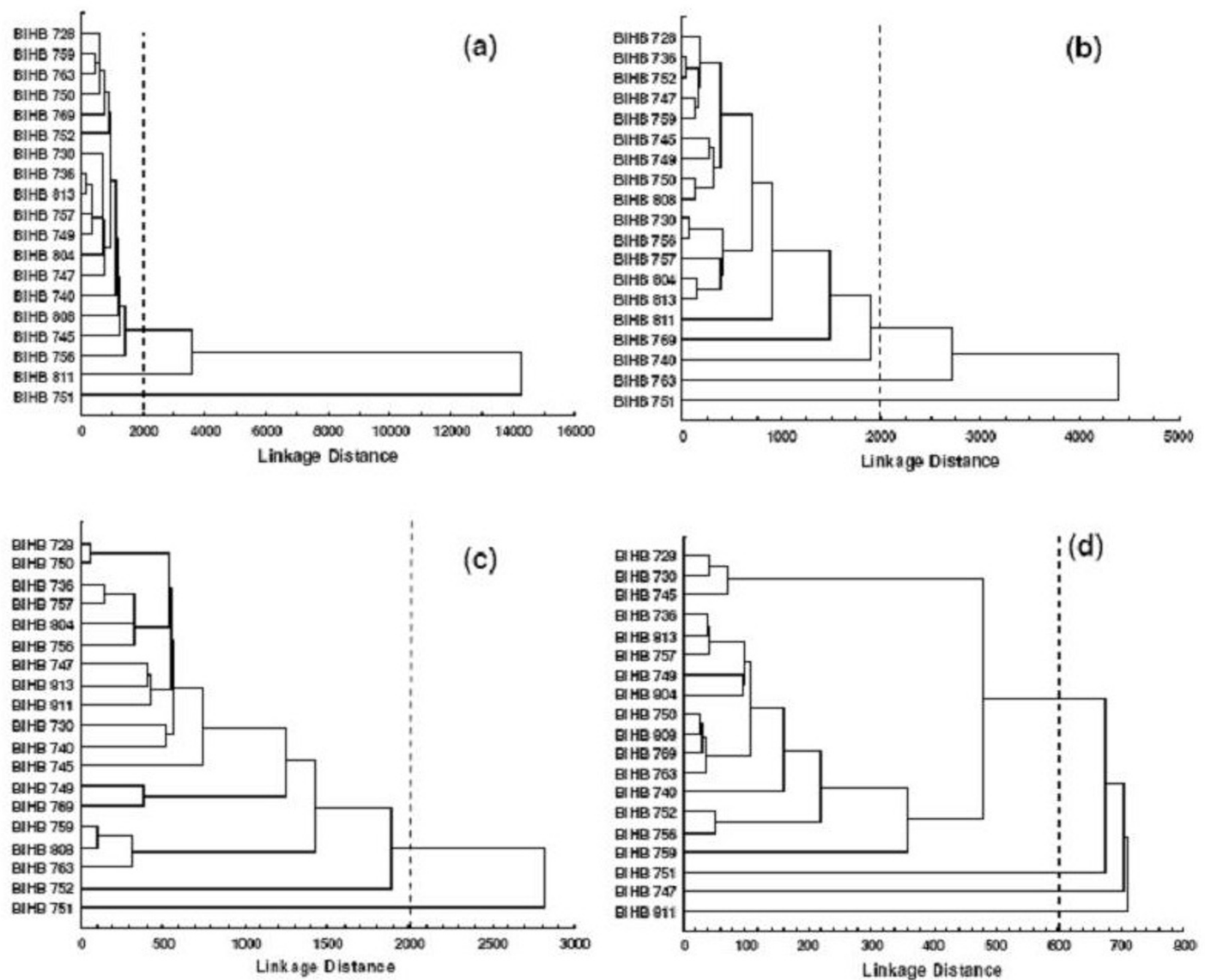

Figure 2

Dendrogram based on organic acid profiles of phosphate-solubilizing fluorescent Pseudomonas grown in NBRIP broth with (a) tricalcium phosphate, (b) Udaipur rock phosphate, (c) Mussoorie rock phosphate, and (d) North Carolina rock phosphate after 5 days incubation at $28^{\circ} \mathrm{C}$.

succinic acid to one strain each of $P$. poae, $P$. fluorescens and Pseudomonas sp.; formic acid to P. fluorescens strain; citric acid to one strain each of $P$. poae and Pseudomonas sp.; and malic acid to one P. trivialis, P. fluorescens and three Pseudomonas spp. strains.

Quantitative difference in the production of organic acids was observed during the solubilization of phosphate substrates by Pseudomonas strains (Tables 2, 3, 4, 5). The quantities of organic acids produced during TCP solubilization ranged from 216.7-19340 $\mu \mathrm{g} / \mathrm{ml}$ gluconic acid, 14.3-532.3 $\mu \mathrm{g} / \mathrm{ml}$ 2-ketogluconic acid, 96-2249 $\mu \mathrm{g} / \mathrm{ml}$ succinic acid, 23.8-132.0 $\mu \mathrm{g} / \mathrm{ml}$ formic acid, 25.5-65.2 $\mu \mathrm{g} / \mathrm{ml}$ citric acid, and 75-4215 $\mu \mathrm{g} / \mathrm{ml}$ malic acid. Lactic acid production shown only by $P$. trivialis BIHB 728 and Pseudomonas sp. BIHB 804 was 53.7 and $49.3 \mu \mathrm{g} / \mathrm{ml}$, respectively. Oxalic acid production detected only for Pseudomonas sp. BIHB 751 was $318.7 \mu \mathrm{g} / \mathrm{ml}$ during TCP solubilization. Organic acid production during URP solubilization varied from $8-26.6 \mu \mathrm{g} / \mathrm{ml}$ oxalic acid, 631.7$10903 \mu \mathrm{g} / \mathrm{ml}$ gluconic acid, 16.4-255 $\mu \mathrm{g} / \mathrm{ml}$ 2-ketogluconic acid, 41.3-164 $\mu \mathrm{g} / \mathrm{ml}$ lactic acid, 56.1-108 $\mu \mathrm{g} / \mathrm{ml}$ succinic acid, and 34.5-4350 $\mathrm{\mu g} / \mathrm{ml}$ malic acid. Formic acid production only by $P$. trivialis BIHB 745 and $P$. trivialis BIHB 763 was 35.1 and $93.6 \mu \mathrm{g} / \mathrm{ml}$, respectively. During MRP solubilization the quantities of organic acids estimated in the culture filtrates were $10.6-39.3 \mu \mathrm{g} / \mathrm{ml}$ oxalic acid, 7076.3-15727 $\mu \mathrm{g} / \mathrm{ml}$ gluconic acid, $18.4-468$ 
$\mu \mathrm{g} / \mathrm{ml}$ 2-ketogluconic acid, 36.8-50.8 $\mu \mathrm{g} / \mathrm{ml}$ lactic acid, $136.0-349.7 \mu \mathrm{g} / \mathrm{ml}$ succinic acid, $70.4-114.4 \mu \mathrm{g} / \mathrm{ml}$ formic acid, and 32.3-2802 $\mu \mathrm{g} / \mathrm{ml}$ malic acid. Citric acid production observed for only Pseudomonas sp. BIHB 811 was $22.3 \mu \mathrm{g} / \mathrm{ml}$ during MRP solubilization. Organic acids during NCRP solubilization ranged from $8.9-17.1 \mu \mathrm{g} / \mathrm{ml}$ oxalic acid, 2549-6035 $\mu \mathrm{g} / \mathrm{ml}$ gluconic acid, 10.1-32.7 $\mu \mathrm{g} / \mathrm{ml}$ 2-ketogluconic acid, 38.8-54.3 $\mu \mathrm{g} / \mathrm{ml}$ lactic acid, $45.1-75.1 \mu \mathrm{g} / \mathrm{ml}$ succinic acid, and 33-415 $\mu \mathrm{g} / \mathrm{ml}$ malic acid. Citric acid production shown by the two strains $P$. poae BIHB 752 and Pseudomonas sp. BIHB 756 was 26.1 and $29.5 \mu \mathrm{g} / \mathrm{ml}$, respectively. Pseudomonas fluorescens BIHB 740 produced $59.3 \mu \mathrm{g} / \mathrm{ml}$ formic acid during NCRP solubilization.

Table 6: Influence of fluorescent Pseudomonas on growth and nutrient content of maize after 90 days in Environment Control Chamber.

\begin{tabular}{|c|c|c|c|c|c|c|c|c|c|c|}
\hline \multirow[b]{3}{*}{ Treatment } & \multicolumn{4}{|c|}{ Growth parameter } & \multicolumn{6}{|c|}{ Nutrient content (\%) } \\
\hline & \multirow[b]{2}{*}{$\begin{array}{l}\text { Plant height } \\
\text { (cm) }\end{array}$} & \multirow[b]{2}{*}{$\begin{array}{l}\text { Shoot DW } \\
\text { (g/plant) }\end{array}$} & \multirow[b]{2}{*}{$\begin{array}{l}\text { Root length } \\
(\mathrm{cm})\end{array}$} & \multirow[b]{2}{*}{$\begin{array}{l}\text { Root DW } \\
\text { (g/plant) }\end{array}$} & \multicolumn{3}{|c|}{ Shoot } & \multicolumn{3}{|c|}{ Root } \\
\hline & & & & & $\mathrm{N}$ & $\mathrm{P}$ & $\mathrm{K}$ & $N$ & $\mathrm{P}$ & $\mathrm{K}$ \\
\hline $\mathrm{NP}_{0} \mathrm{~K}$ & $116 . \mathrm{I}^{\mathrm{h}}$ & $4.03^{f}$ & $17.5 \mathrm{~g}$ & $0.47 \mathrm{hi}$ & $1.83^{d}$ & 0.18 & $2.50^{\mathrm{ef}}$ & $\mathrm{I} .39 \mathrm{~g}$ & $0.08^{i}$ & $0.6 \mathrm{I}^{\mathrm{d}}$ \\
\hline $\mathrm{NP}_{\mathrm{TCP}} \mathrm{K}$ & $126.4^{\mathrm{fgh}}$ & $4.38^{\mathrm{ef}}$ & $18.5 \mathrm{fg}$ & $0.55^{\mathrm{hi}}$ & $1.95^{\mathrm{cd}}$ & $0.24 \mathrm{ij}$ & $2.37^{f}$ & $1.40 \mathrm{fg}$ & $0.14^{\mathrm{hi}}$ & $0.65^{\mathrm{cd}}$ \\
\hline $\mathrm{NP}_{\mathrm{SSP}} \mathrm{K}$ & $135.5^{b c d e f}$ & $4.6 \mathrm{I}_{\mathrm{ef}}$ & $20.3^{\text {efg }}$ & $0.88^{\mathrm{de}}$ & $1.98 \mathrm{~cd}$ & $\left.0.3\right|_{\text {hij }}$ & $2.63^{\mathrm{cdef}}$ & $1.43^{\mathrm{efg}}$ & $0.25^{\text {defg }}$ & $0.70^{\mathrm{cd}}$ \\
\hline $\begin{array}{l}\mathrm{NP}_{\mathrm{TCP}} \mathrm{K}+\mathrm{Pt} \\
\mathrm{BIHB}_{7} \mathrm{~T}\end{array}$ & $|3| . \mid$ cdefg & ${ }^{4.84} \mathrm{ef}$ & 20.9 defg & $0.64 \mathrm{gh}$ & $1.95^{\mathrm{cd}}$ & 0.37 efghi & $2.67 \mathrm{cdef}$ & $1.97 \mathrm{ab}$ & $0.26^{\text {defg }}$ & $0.93^{\mathrm{ab}}$ \\
\hline $\begin{array}{l}\mathrm{NP}_{\mathrm{TCP}} \mathrm{K}+\mathrm{Pt} \\
\mathrm{BIHB} 736\end{array}$ & $130.0^{\mathrm{efg}}$ & $4.5 \mathrm{I}^{\mathrm{ef}}$ & $27.1^{\mathrm{a}}$ & $0.55^{\mathrm{hi}}$ & $2.22^{\mathrm{abcd}}$ & $0.34 \mathrm{ghi}$ & $3.13^{\text {abcde }}$ & $2.03^{a}$ & $0.21 \mathrm{gh}$ & $0.85^{\mathrm{abc}}$ \\
\hline $\begin{array}{l}\mathrm{NP}_{\mathrm{TCP}} \mathrm{K}+\mathrm{Pt} \\
\mathrm{BIHB} 745\end{array}$ & $145.9 \mathrm{ab}$ & $7.57 \mathrm{abc}$ & $26.6^{\mathrm{ab}}$ & $1.16^{\mathrm{b}}$ & $2.72^{\mathrm{ab}}$ & $0.64^{\mathrm{a}}$ & $3.43^{\mathrm{ab}}$ & $1.9 \mathrm{I} a \mathrm{abc}$ & $0.40^{a}$ & $0.98^{a}$ \\
\hline $\begin{array}{l}\mathrm{NP}_{\mathrm{TCP}} \mathrm{K}+\mathrm{Pt} \\
\mathrm{BIHB} 747\end{array}$ & 142.0abcde & 7.79ab & $24.8^{\mathrm{abcd}}$ & $\left.1.1\right|^{b c}$ & $2.63^{a b c}$ & $0.56 \mathrm{abc}$ & $3.10^{\text {abcde }}$ & I.84abcde & $0.32^{\text {bcde }}$ & $0.86 \mathrm{abc}$ \\
\hline $\begin{array}{l}\mathrm{NP}_{\mathrm{TCP}} \mathrm{K}+\mathrm{Pt} \\
\mathrm{BIHB} 749\end{array}$ & $14 \mid .5^{\text {abcde }}$ & 6.04 bcde & $24.9 \mathrm{abcd}$ & $1.34^{\mathrm{a}}$ & $2.20^{\mathrm{abcd}}$ & $0.43^{\text {cdefgh }}$ & $2.92^{\mathrm{bcdef}}$ & $1.50^{\text {cdefg }}$ & $0.23^{f g}$ & $0.74 \mathrm{bcd}$ \\
\hline $\begin{array}{l}\mathrm{NP}_{\mathrm{TCP}} \mathrm{K}+\mathrm{Pt} \\
\mathrm{BIHB} 750\end{array}$ & $126.8^{\mathrm{fgh}}$ & $4.75^{\mathrm{ef}}$ & 20.9 defg & $0.5 \mathrm{I}^{\mathrm{hi}}$ & $2.18^{\mathrm{abcd}}$ & $0.57^{a b c}$ & $2.60^{\text {def }}$ & $1.55^{\text {bcdefg }}$ & $\left.0.3\right|^{\text {cde }}$ & $0.74^{b c d}$ \\
\hline $\begin{array}{l}\mathrm{NP}_{\mathrm{TCP}} \mathrm{K}+\mathrm{Pt} \\
\mathrm{BIHB} 757\end{array}$ & $142.6^{\mathrm{abcd}}$ & $5.63 \mathrm{def}$ & $23.5^{\mathrm{abcd}}$ & $1.08^{b c}$ & $2.45^{\mathrm{abcd}}$ & $0.50^{\mathrm{abcdef}}$ & $2.83^{\mathrm{bcdef}}$ & $1.63^{\mathrm{abcdefg}}$ & $0.24^{\mathrm{efg}}$ & 0.79 abcd \\
\hline $\begin{array}{l}\mathrm{NP}_{\mathrm{TCP}} \mathrm{K}+\mathrm{Pt} \\
\mathrm{BIHB} 759\end{array}$ & $148.8^{a}$ & $5.14^{\mathrm{def}}$ & $25.8^{a b c}$ & $0.62^{\mathrm{gh}}$ & $2.49 \mathrm{abcd}$ & $0.53^{\mathrm{abcd}}$ & $3.47^{\mathrm{ab}}$ & $1.93^{\mathrm{ab}}$ & $0.30^{\text {cdef }}$ & $0.74 \mathrm{bcd}$ \\
\hline $\begin{array}{l}\mathrm{NP}_{\mathrm{TCP}} \mathrm{K}+\mathrm{Pt} \\
\mathrm{BIHB} 763\end{array}$ & $146.0^{\mathrm{ab}}$ & $4.82^{\mathrm{ef}}$ & $24.0^{\mathrm{abcd}}$ & $0.66 \mathrm{fgh}^{\mathrm{g}}$ & $2.60^{a b c}$ & $0.49 \mathrm{bcdefg}$ & $2.93 \mathrm{bcdef}$ & 1.70abcdefg & $0.26^{\mathrm{defg}}$ & $0.83^{a b c d}$ \\
\hline $\begin{array}{l}\mathrm{NP}_{\mathrm{TCP}} \mathrm{K}+\mathrm{Pt} \\
\mathrm{BIHB} 769\end{array}$ & |4|.0abcde & $7.70^{a b c}$ & $26.5^{\mathrm{ab}}$ & 0.84 def & $2.10^{\mathrm{bcd}}$ & 0.39defgh & 2.60 def & $1.56^{b c d e f g}$ & $0.23^{\mathrm{fg}}$ & $0.74 \mathrm{bcd}$ \\
\hline $\begin{array}{l}N_{\text {TCP }} \mathrm{K}+P P \\
\mathrm{BIHB} 730\end{array}$ & $126.4^{\mathrm{fgh}}$ & $8.55 a$ & $26.5^{\mathrm{ab}}$ & $\left.0.8\right|^{\text {efg }}$ & $2.27 \mathrm{abcd}$ & $\left.0.5\right|^{\text {abcde }}$ & $2.77^{\mathrm{cdef}}$ & I.49cdefg & $0.25^{\text {defg }}$ & $0.74 b c d$ \\
\hline $\begin{array}{l}N_{\text {TCP }} \mathrm{K}+P p \\
\mathrm{BIHB} 752\end{array}$ & 130.6 defg & 5.89 cdef & $22.4^{\text {bcdef }}$ & $0.52^{\mathrm{hi}}$ & $2.15^{\mathrm{bcd}}$ & 0.36 fghi & $3.27 a b c$ & $1.95^{\mathrm{ab}}$ & $0.39 \mathrm{ab}$ & $0.78^{a b c d}$ \\
\hline $\begin{array}{l}\mathrm{NP}_{\mathrm{TCP}} \mathrm{K}+\mathrm{Pp} \\
\mathrm{BIHB} 808\end{array}$ & $143.5^{\mathrm{abc}}$ & $5.46 \mathrm{def}$ & 24. I abcd & $0.63 \mathrm{gh}$ & $2.64^{a b c}$ & $0.63^{\mathrm{ab}}$ & 3.10abcde & $1.88 \mathrm{abcd}$ & $0.27^{c d e f}$ & $0.68 \mathrm{cdcd}$ \\
\hline $\begin{array}{l}\mathrm{NP}_{\mathrm{TCP}} \mathrm{K}+\mathrm{Pf} \\
\mathrm{BIHB} 740\end{array}$ & 137.0abcdefg & $6.83^{\mathrm{abcd}}$ & $24.8^{\mathrm{abcd}}$ & $1.0 \mathrm{lbcd}$ & $2.58^{\mathrm{abc}}$ & 0.39 defgh & $2.75^{\mathrm{cdef}}$ & $1.43^{\mathrm{efg}}$ & $0.24^{\mathrm{defg}}$ & $0.82^{\mathrm{abcd}}$ \\
\hline $\begin{array}{l}\mathrm{NP}_{\mathrm{TCP}} \mathrm{K}+\mathrm{P}_{\mathrm{SP}} \\
\mathrm{BIHB} 75 \mathrm{I}\end{array}$ & $119.5 \mathrm{gh}$ & $4.84 \mathrm{ef}^{-}$ & $22.5^{\mathrm{bcdef}}$ & $0.4 \mathrm{I}^{\mathrm{i}}$ & $2.58^{a b c}$ & $0.30^{\text {hij }}$ & $2.72^{\mathrm{cdef}}$ & 1.47defg & $0.20 \mathrm{gh}$ & $0.62^{d}$ \\
\hline $\begin{array}{l}\mathrm{NP}_{\mathrm{TCP}} \mathrm{K}+\mathrm{P}_{\mathrm{SP}} \\
\mathrm{BIHB} 756\end{array}$ & $|4| . \mid$ abcde & 6.88abcd & $26.0^{\mathrm{ab}}$ & $0.92^{\text {cde }}$ & $2.88^{a}$ & $\left.0.6\right|^{\mathrm{ab}}$ & $3.67^{\mathrm{a}}$ & $1.90 \mathrm{abc}$ & $0.35^{a b c}$ & $0.82^{\mathrm{abcd}}$ \\
\hline $\begin{array}{l}\mathrm{NP}_{\mathrm{TCP}} \mathrm{K}+\mathrm{P}_{\mathrm{SP}} \\
\mathrm{BIHB} 804\end{array}$ & $|3| .4^{\text {cdefg }}$ & $5.03 \mathrm{def}$ & $23.4^{\mathrm{abcd}}$ & 0.96 cde & $2.40^{\mathrm{abcd}}$ & $0.59 \mathrm{ab}$ & 3.17 $7 \mathrm{abcd}$ & $1.37 \mathrm{~g}$ & $0.20 \mathrm{gh}$ & $0.79 \mathrm{abcd}$ \\
\hline $\begin{array}{l}\mathrm{NP}_{\mathrm{TCP}} \mathrm{K}+\mathrm{P}_{\mathrm{sp}} \\
\mathrm{BIHB} 8 \mathrm{II}\end{array}$ & $127.3^{\mathrm{fgh}}$ & $4.46^{\mathrm{ef}}$ & $18.5^{f g}$ & $0.58^{\mathrm{hi}}$ & $2.25^{\mathrm{abcd}}$ & $0.3 I^{\text {hij }}$ & $2.63^{\mathrm{cdef}}$ & $1.95^{\mathrm{ab}}$ & $0.32^{\mathrm{bcd}}$ & $0.77^{b c d}$ \\
\hline $\begin{array}{l}\mathrm{NP}_{\mathrm{TCP}} \mathrm{K}+\mathrm{P}_{\mathrm{SP}} \\
\mathrm{BIHB} 813\end{array}$ & 130.9defg & $8.58^{a}$ & $21.4^{\mathrm{cdefg}}$ & $0.48^{\mathrm{hi}}$ & $2.47^{a b c d}$ & 0.39 defgh & $3.27 \mathrm{abc}$ & $1.82^{\text {abcdefg }}$ & $0.22 \mathrm{gh}$ & $0.76^{\mathrm{bcd}}$ \\
\hline
\end{tabular}

Values are the mean of 8 replicates. N and K applied as ammonium sulfate @ $240 \mathrm{~kg} \mathrm{~N} / \mathrm{ha}$, and muriate of potash @ $80 \mathrm{~kg} \mathrm{~K} / \mathrm{ha}$ to all the treatments, respectively. TCP $=$ tricalcium phosphate $(120 \mathrm{~kg} \mathrm{P} / \mathrm{ha}) . \mathrm{SSP}=$ single super phosphate $(120 \mathrm{~kg} P / \mathrm{ha})$. Values with common letters in each column do not differ statistically according to Duncan's Multiple Range Test at $\mathrm{P} \leq 0.0 \mathrm{I}$. $\mathrm{DW}=$ dry weight, $P t=P$. trivialis, $P p=P$. poae, $P f=P$. fluorescens, and Psp $=$ Pseudomonas 
Cluster analysis based on the organic acid profiles during TCP, URP, MRP and NCRP solubilization generated Pseudomonas groups with strains belonging to the same or different species (Fig. 2). For TCP solubilization a single cluster was obtained at 2000 linkage distance, while Pseudomonas sp. BIHB 751 and Pseudomonas sp. BIHB 811 stood outside the cluster (Fig. 2a). Pseudomonas sp. BIHB 751 differed from the other strains in producing oxalic acid, lack of succinic acid production, and producing the lowest quantity of gluconic acid and the highest quantity of 2-ketogluconic acid. Pseudomonas sp. BIHB 811 showed dissimilarity in not producing malic acid. In URP solubilization a single cluster of three sub-clusters and single branches of Pseudomonas sp. BIHB 811, P. trivialis BIHB 769 and $P$. fluorescens BIHB 740 were formed at 2000 linkage distance, while Pseudomonas sp. BIHB 751 and P. trivialis BIHB 763 stood independently outside the cluster (Fig. 2b). Pseudomonas sp. BIHB 751 differed in producing the lowest quantity of gluconic acid and the highest quantities of 2-ketogluconic and malic acids. Pseudomonas trivialis BIHB 763 was separate from other strains in producing the highest quantities of gluconic and formic acids (Fig. 2b). During MRP solubilization a single cluster including six sub-clusters and two single branches of $P$. trivialis BIHB 745 and $P$. poae BIHB 752 were observed at 2000 linkage distance. Pseudomonas sp. BIHB 751 stood separately outside the cluster in producing the lowest quantity of gluconic acid and the highest quantity of malic acid (Fig. 2c). In NCRP solubilization P. trivialis BIHB 747, Pseudomonas sp. BIHB 751 and Pseudomonas sp. BIHB 811 stood outside the cluster as independent branches at 600 linkage distance (Fig 2d). The cluster incorporated 5 sub-clusters and separate branches of Pseudomonas sp. BIHB 740 and P. trivialis BIHB 759. Pseudomonas trivialis BIHB 747 differed in the highest gluconic acid production, Pseudomonas sp. BIHB 751 in the highest malic acid production, and Pseudomonas sp. BIHB 811 in producing the lowest quantity of gluconic acid and the highest quantity of 2-ketogluconic, lactic, and succinic acids.

\section{Influence on plant growth}

Significant difference was observed for the growth parameters in maize among PSB treatments and uninoculated control treatments (Table 6). The plant height was significantly higher in fifteen PSB treatments and $\mathrm{NP}_{\mathrm{SSP}} \mathrm{K}$ over $\mathrm{NP}_{0} \mathrm{~K}$. Increment in height was significantly higher with $\mathrm{NP}_{\mathrm{TCP}} \mathrm{K}+P t$ BIHB 759 and significantly lower with $\mathrm{NP}_{\mathrm{TCP}} \mathrm{K}+$ Psp BIHB 751 over $\mathrm{NP}_{\mathrm{SSP}} \mathrm{K}$. Other PSB treatments were statistically at par with $\mathrm{NP}_{\mathrm{SSP}} \mathrm{K}$. Nine PSB treatments exhibited significantly higher plant height over $\mathrm{NP}_{\mathrm{TCP}} \mathrm{K}$. Plant height increase was the highest with $\mathrm{NP}_{\mathrm{TCP}} \mathrm{K}+P t$ BIHB 759, followed by $\mathrm{NP}_{\mathrm{TCP}} \mathrm{K}+P t$ BIHB 763, $\mathrm{NP}_{\mathrm{TCP}} \mathrm{K}+P t$ BIHB 745, $\mathrm{NP}_{\mathrm{TCP}} \mathrm{K}+P p$ BIHB 808, $\mathrm{NP}_{\mathrm{TCP}} \mathrm{K}+P t$ BIHB 757, and $\mathrm{NP}_{\mathrm{TCP}} \mathrm{K}+$ Pt BIHB 747 treatments.
The shoot dry weight was significantly higher in seven PSB treatments over $\mathrm{NP}_{0} \mathrm{~K}, \mathrm{NP}_{\mathrm{TCP}} \mathrm{K}$ and $\mathrm{NP}_{\mathrm{SSP}} \mathrm{K}$. The highest shoot dry weight with $\mathrm{NP}_{\mathrm{TCP}} \mathrm{K}+$ Psp BIHB 813 was statistically at par with $\mathrm{NP}_{\mathrm{TCP}} \mathrm{K}+P p$ BIHB 730, $\mathrm{NP}_{\mathrm{TCP}} \mathrm{K}+P t$ BIHB 747, $\mathrm{NP}_{\mathrm{TCP}} \mathrm{K}+P t$ BIHB 769, $\mathrm{NP}_{\mathrm{TCP}} \mathrm{K}+P t$ BIHB 745, $\mathrm{NP}_{\mathrm{TCP}} \mathrm{K}+$ Psp BIHB 756 and $\mathrm{NP}_{\mathrm{TCP}} \mathrm{K}+P f$ BIHB 740. The root length was significantly higher in fifteen PSB treatments over $\mathrm{NP}_{0} \mathrm{~K}$ and thirteen PSB treatments over $\mathrm{NP}_{\mathrm{TCP}} \mathrm{K}$ and $\mathrm{NP}_{\mathrm{SSP}} \mathrm{K}$. The maximum increase was obtained with $\mathrm{NP}_{\mathrm{TCP}} \mathrm{K}+$ Pt $\mathrm{BIHB} 736$, followed by $\mathrm{NP}_{\mathrm{TCP}} \mathrm{K}+P t$ BIHB 745, $\mathrm{NP}_{\mathrm{TCP}} \mathrm{K}+$ Pt BIHB 769, $\mathrm{NP}_{\mathrm{TCP}} \mathrm{K}+P p$ BIHB 730 and $\mathrm{NP}_{\mathrm{TCP}} \mathrm{K}+P$ sp BIHB 756. The treatments $\mathrm{NP}_{\mathrm{TCP}} \mathrm{K}$ and $\mathrm{NP}_{\mathrm{SSP}} \mathrm{K}$ were statistically at par with $\mathrm{NP}_{0} \mathrm{~K}$. The root dry weight was significantly higher in $\mathrm{NP}_{\mathrm{TCP}} \mathrm{K}+P t$ BIHB 749 over other PSB treatments, $\mathrm{NP}_{0} \mathrm{~K}, \mathrm{NP}_{\mathrm{TCP}} \mathrm{K}$ and $\mathrm{NP}_{\mathrm{SSP}} \mathrm{K}$. The treatments $\mathrm{NP}_{\mathrm{TCP}} \mathrm{K}+P t$ BIHB 745, $\mathrm{NP}_{\mathrm{TCP}} \mathrm{K}+P t$ BIHB 747 and $\mathrm{NP}_{\mathrm{TCP}} \mathrm{K}+P t$ BIHB 757 were statistically at par and showed significantly higher root dry weight over $\mathrm{NP}_{0} \mathrm{~K}$, $\mathrm{NP}_{\mathrm{TCP}} \mathrm{K}$ and $\mathrm{NP}_{\mathrm{SSP}} \mathrm{K}$.

\section{Plant NPK content}

The treatments showed significant difference in the nutrient content of roots and shoots (Table 6). The shoot $\mathrm{N}$ was statistically higher in seven PSB treatments over $\mathrm{NP}_{0} \mathrm{~K}$ and two PSB treatments over $\mathrm{NP}_{0} \mathrm{~K}, \mathrm{NP}_{\mathrm{TCP}} \mathrm{K}$ and $\mathrm{NP}_{\mathrm{SSP}} \mathrm{K}$. A non-significant difference in the shoot $\mathrm{N}$ was observed with $\mathrm{NP}_{0} \mathrm{~K}, \mathrm{NP}_{\mathrm{TCP}} \mathrm{K}$ and $\mathrm{NP}_{\mathrm{SSP}} \mathrm{K}$. The shoot $\mathrm{P}$ was significantly higher in ten PSB treatments over $\mathrm{NP}_{0} \mathrm{~K}, \mathrm{NP}_{\mathrm{TCP}} \mathrm{K}$ and $\mathrm{NP}_{\mathrm{SSP}} \mathrm{K}$. The highest $\mathrm{P}$ content obtained with $\mathrm{NP}_{\mathrm{TCP}} \mathrm{K}+$ Pt BIHB 745. The treatments $\mathrm{NP}_{\mathrm{TCP}} \mathrm{K}$ and $\mathrm{NP}_{\mathrm{SSP}} \mathrm{K}$ were statistically at par with $\mathrm{NP}_{0} \mathrm{~K}$. The shoot $\mathrm{K}$ was significantly higher in $\mathrm{NP}_{\mathrm{TCP}} \mathrm{K}+P s p$ BIHB 756, $\mathrm{NP}_{\mathrm{TCP}} \mathrm{K}+P t \mathrm{BIHB}$ 759 and $\mathrm{NP}_{\mathrm{TCP}} \mathrm{K}+$ Pt $\mathrm{BIHB} 745$ over $\mathrm{NP}_{0} \mathrm{~K}, \mathrm{NP}_{\mathrm{TCP}} \mathrm{K}$ and $\mathrm{NP}_{\mathrm{SSP}} \mathrm{K}$.

The root $\mathrm{N}$ was significantly higher in eight PSB treatments over $\mathrm{NP}_{0} \mathrm{~K}, \mathrm{NP}_{\mathrm{TCP}} \mathrm{K}$ and $\mathrm{NP}_{\mathrm{SSP}} \mathrm{K}$. The $\mathrm{N}$ content was statistically at par in $\mathrm{NP}_{0} \mathrm{~K}, \mathrm{NP}_{\mathrm{TCP}} \mathrm{K}$ and $\mathrm{NP}_{\mathrm{SSP}} \mathrm{K}$. The highest $\mathrm{N}$ was obtained with $\mathrm{NP}_{\mathrm{TCP}} \mathrm{K}+P t$ BIHB 736. The root $\mathrm{P}$ was significantly higher in three PSB treatments over $\mathrm{NP}_{\mathrm{SSP}} \mathrm{K}$. The maximum increase was obtained with $\mathrm{NP}_{\mathrm{TCP}} \mathrm{K}+P t$ BIHB 745, followed by $\mathrm{NP}_{\mathrm{TCP}} \mathrm{K}+P p$ BIHB 752 and $\mathrm{NP}_{\mathrm{TCP}} \mathrm{K}+P$ sp $\mathrm{BIHB}$ 756. The $\mathrm{P}$ content was significantly higher in $\mathrm{NP}_{\mathrm{SSP}} \mathrm{K}$ over $\mathrm{NP}_{0} \mathrm{~K}$ and $\mathrm{NP}_{\mathrm{TCP}} \mathrm{K}$. The root $\mathrm{K}$ was significantly higher in $\mathrm{NP}_{\mathrm{TCP}} \mathrm{K}+$ Pt $\mathrm{BIHB} 745$ and $\mathrm{NP}_{\mathrm{TCP}} \mathrm{K}+$ Pt BIHB 728 over $\mathrm{NP}_{0} \mathrm{~K}, \mathrm{NP}_{\mathrm{TCP}} \mathrm{K}$ and $\mathrm{NP}_{\mathrm{SSP}} \mathrm{K}$. Other treatments were statistically at par with $\mathrm{NP}_{\mathrm{TCP}} \mathrm{K}$ and $\mathrm{NP}_{\mathrm{SSP}} \mathrm{K}$.

\section{Soil properties}

The soil pH, organic matter and available N, P, K contents were significantly affected by PSB treatments (Table 7). The final $\mathrm{pH}$ with non-significant difference among various treatments was less than the initial $\mathrm{pH}$. The highest decrease recorded with $\mathrm{NP}_{\mathrm{TCP}} \mathrm{K}+P t$ BIHB 757 was statisti- 
Table 7: Influence of fluorescent Pseudomonas on soil properties after 90 days in maize in Environment Control Chamber.

\begin{tabular}{|c|c|c|c|c|c|c|}
\hline \multirow[b]{2}{*}{ Treatment } & \multirow[b]{2}{*}{$\mathrm{pH}$} & \multirow[b]{2}{*}{ OM (\%) } & \multicolumn{4}{|c|}{ Available nutrients (\%) } \\
\hline & & & $\mathrm{N}$ & $P$ & K & $\mathrm{Ca}$ \\
\hline $\mathrm{NP}_{0} \mathrm{~K}$ & $6.73^{a}$ & $3.40 \mathrm{ghi}$ & $0.044^{\mathrm{hij}}$ & $0.0015^{\mathrm{kl}}$ & 0.020 fgh & $0.032^{i}$ \\
\hline $\mathrm{NP}_{\mathrm{TCP}} \mathrm{K}$ & $6.63^{\mathrm{ab}}$ & $3.63^{\text {defghi }}$ & 0.049 efgh & 0.002 Ighij & $0.025^{\text {cde }}$ & $0.038^{\mathrm{h}}$ \\
\hline $\mathrm{NP}_{\mathrm{SSP}} \mathrm{K}$ & $6.50^{\mathrm{abc}}$ & $3.48^{\text {efghi }}$ & 0.046 fghi & $0.0025^{\text {defg }}$ & $0.022^{\mathrm{efg}}$ & $0.033^{\mathrm{hi}}$ \\
\hline $\mathrm{NP}_{\mathrm{TCP}} \mathrm{K}+\mathrm{Pt}_{t} \mathrm{BIHB} 728$ & $6.26^{\mathrm{abcd}}$ & $3.90^{\text {bcde }}$ & $0.052^{\mathrm{def}}$ & $0.0019 \mathrm{ijkl}$ & $0.025^{\text {cde }}$ & $0.069 \mathrm{bc}$ \\
\hline $\mathrm{NP}_{\mathrm{TCP}} \mathrm{K}+\mathrm{Pt}_{t} \mathrm{BIHB} 736$ & $6.23^{\mathrm{bcd}}$ & $3.42^{\text {fghi }}$ & $0.057^{\mathrm{bcd}}$ & $0.0026^{\text {defg }}$ & 0.024 def & $0.057 \mathrm{fg}$ \\
\hline $\mathrm{NP}_{\mathrm{TCP}} \mathrm{K}+\mathrm{Pt}$ BIHB 745 & $5.93^{d}$ & $4.17^{\mathrm{ab}}$ & $0.065^{\mathrm{a}}$ & $0.0038^{a}$ & $0.033^{\mathrm{ab}}$ & $0.085^{a}$ \\
\hline $\mathrm{NP}_{\mathrm{TCP}} \mathrm{K}+\mathrm{Pt}_{t} \mathrm{BIHB} 747$ & $6.02^{\mathrm{cd}}$ & $4.13^{\mathrm{abc}}$ & $0.062^{\mathrm{ab}}$ & $0.0027^{c d e f}$ & $0.030 \mathrm{abc}$ & $0.08 \mathrm{I}^{\mathrm{a}}$ \\
\hline $\mathrm{NP}_{\mathrm{TCP}} \mathrm{K}+\mathrm{Pt}$ BIHB 749 & $6.12^{\mathrm{cd}}$ & 3.57 efghi & $0.042^{\mathrm{ijk}}$ & $0.0024^{\text {efgh }}$ & $0.029 \mathrm{bc}$ & $0.074 b$ \\
\hline $\mathrm{NP}_{\mathrm{TCP}} \mathrm{K}+\mathrm{Pt}_{t} \mathrm{BIHB} 750$ & $6.24 \mathrm{bcd}$ & $3.55^{\text {efghi }}$ & $0.039 \mathrm{jkl}$ & $0.0019 \mathrm{jkkl}$ & $0.019 \mathrm{fgh}$ & $0.080^{a}$ \\
\hline $\mathrm{NP}_{\mathrm{TCP}} \mathrm{K}+\mathrm{Pt}_{t} \mathrm{BIHB} 757$ & $5.93^{d}$ & 3.79bcdefg & $0.059 \mathrm{bc}$ & $0.0024^{\text {efgh }}$ & 0.026 cde & $0.070^{\mathrm{bc}}$ \\
\hline $\mathrm{NP}_{\mathrm{TCP}} \mathrm{K}+\mathrm{Pt}_{t} \mathrm{BIHB} 759$ & $6.20 \mathrm{bcd}$ & $4.00^{\mathrm{abcd}}$ & $0.040 \mathrm{jk}$ & $0.0022^{\text {fghi }}$ & $0.022^{\text {efgh }}$ & $0.072^{b}$ \\
\hline $\mathrm{NP}_{\mathrm{TCP}} \mathrm{K}+\mathrm{Pt} \mathrm{BIHB} 763$ & $6.18^{\mathrm{bcd}}$ & $3.82^{\mathrm{bcdefg}}$ & $0.039 \mathrm{kl}$ & 0.0028 cde & $0.018^{\mathrm{gh}}$ & $0.058^{\mathrm{ef}}$ \\
\hline $\mathrm{NP}_{\mathrm{TCP}} \mathrm{K}+\mathrm{Pt}_{t} \mathrm{BIHB} 769$ & $6.30^{\mathrm{abcd}}$ & $3.29 i$ & $0.046 \mathrm{ghi}$ & 0.0026 cdef & 0.027 cde & $0.059 \mathrm{e}$ \\
\hline $\mathrm{NP}_{\mathrm{TCP}} \mathrm{K}+\mathrm{Pp} \mathrm{BIHB} 730$ & $6.23 \mathrm{bcd}$ & $3.55^{\text {efghi }}$ & $0.050^{\mathrm{efg}}$ & $0.0020^{\mathrm{hijkl}}$ & 0.027 cde & $0.052 \mathrm{~g}$ \\
\hline $\mathrm{NP}_{\mathrm{TCP}} \mathrm{K}+\mathrm{Pp} \mathrm{BlHB} 752$ & $6.17 \mathrm{bcd}$ & $3.89 \mathrm{bcde}$ & $0.037 \mathrm{kl}$ & $0.0020^{\mathrm{hijk}}$ & $0.018^{\mathrm{gh}}$ & $0.057^{f g}$ \\
\hline $\mathrm{NP}_{\mathrm{TCP}} \mathrm{K}+\mathrm{Pp} \mathrm{BIHB} 808$ & $6.21^{\mathrm{bcd}}$ & $3.43^{f g h i}$ & $0.049 \mathrm{fgh}$ & $0.0017^{\mathrm{ijk}}$ & $0.022^{\text {efg }}$ & $0.06 / \mathrm{de}$ \\
\hline $\mathrm{NP}_{\mathrm{TCP}} \mathrm{K}+\mathrm{Pf} \mathrm{BIHB} 740$ & $6.25 \mathrm{bcd}$ & $3.85^{\mathrm{bcdef}}$ & $0.055^{\mathrm{cde}}$ & 0.002 Ighij & 0.027 cde & $0.072^{b}$ \\
\hline $\mathrm{NP}_{\mathrm{TCP}} \mathrm{K}+P_{\mathrm{sP}} \mathrm{BIHB} 75 \mathrm{I}$ & $6.33^{\mathrm{abcd}}$ & $3.43 \mathrm{fghi}$ & 0.0341 & $0.0016 \mathrm{jkl}$ & $0.017^{\mathrm{h}}$ & $0.053^{f g}$ \\
\hline $\mathrm{NP}_{\mathrm{TCP}} \mathrm{K}+P_{\mathrm{SP}} \mathrm{BIHB} 756$ & $6.13^{\mathrm{bcd}}$ & $4.32^{\mathrm{a}}$ & $0.060^{\mathrm{abc}}$ & $0.0033^{b}$ & $0.035^{a}$ & $0.072^{b}$ \\
\hline $\mathrm{NP}_{\mathrm{TCP}} \mathrm{K}+P_{\mathrm{sp}} \mathrm{BlHB} 804$ & $6.18^{\mathrm{bcd}}$ & $3.74^{\text {cdefgh }}$ & 0.049 efgh & $0.0015^{1}$ & $0.028^{b c d}$ & $0.069 \mathrm{bc}$ \\
\hline $\mathrm{NP}_{\mathrm{TCP}} \mathrm{K}+P_{\mathrm{SP}} \mathrm{BIHB} 8 \mathrm{II}$ & $6.19 \mathrm{bcd}$ & $4.06^{\mathrm{abc}}$ & $\left.0.05\right|^{\text {efg }}$ & $0.003 I^{b c}$ & $0.022^{\text {efg }}$ & $0.062^{\mathrm{de}}$ \\
\hline $\mathrm{NP}_{\mathrm{TCP}} \mathrm{K}+P_{\mathrm{sP}} \mathrm{BIHB} 8 \mathrm{I} 3$ & $6.17 \mathrm{bcd}$ & $3.36^{\mathrm{hi}}$ & 0.049 fgh & $0.0030^{\mathrm{bcd}}$ & $0.025^{\text {cde }}$ & $0.065^{\mathrm{cd}}$ \\
\hline
\end{tabular}

Values are the mean of 8 replicates. $\mathrm{N}$ and K applied as ammonium sulfate @ $240 \mathrm{~kg} \mathrm{~N} / \mathrm{ha}$, and muriate of potash @ $80 \mathrm{~kg} \mathrm{K/ha}$ to all the treatments, respectively. TCP = tricalcium phosphate $(I 20 \mathrm{~kg} / \mathrm{ha}) . \mathrm{SSP}=$ single super phosphate $(I 20 \mathrm{~kg} P / \mathrm{ha})$. Values with common letters in each column do not differ statistically according to Duncan's Multiple Range Test at $\mathrm{P} \leq 0.0 \mathrm{I}$. $P t=P$. trivialis, $P p=P$. poae, $P f=P$. fluorescens, and $P s p=$ Pseudomonas sp.

cally at par with all other PSB treatments but significantly lower than $\mathrm{NP}_{0} \mathrm{~K}, \mathrm{NP}_{\mathrm{TCP}} \mathrm{K}$ and $\mathrm{NP}_{\mathrm{SSP}} \mathrm{K}$. The organic matter content was significantly higher in four PSB treatments than $\mathrm{NP}_{0} \mathrm{~K}, \mathrm{NP}_{\mathrm{TCP}} \mathrm{K}$ and $\mathrm{NP}_{\mathrm{SSP}} \mathrm{K}$.

The soil $\mathrm{N}$ content was significantly higher in five PSB treatments than $\mathrm{NP}_{0} \mathrm{~K}, \mathrm{NP}_{\mathrm{TCP}} \mathrm{K}$ and $\mathrm{NP}_{\mathrm{SSP}} \mathrm{K}$ and statistically at par among $\mathrm{NP}_{0} \mathrm{~K}, \mathrm{NP}_{\mathrm{TCP}} \mathrm{K}$ and $\mathrm{NP}_{\mathrm{SSP}} \mathrm{K}$. The soil $\mathrm{P}$ content was significantly higher in three PSB treatments over $\mathrm{NP}_{0} \mathrm{~K}, \mathrm{NP}_{\mathrm{TCP}} \mathrm{K}$ and $\mathrm{NP}_{\mathrm{SSP}} \mathrm{K}$. The highest available $\mathrm{P}$ content was obtained with $\mathrm{NP}_{\mathrm{TCP}} \mathrm{K}+\mathrm{Pt}$ BIHB745 among PSB treatments and with $\mathrm{NP}_{S S P} \mathrm{~K}$ among uninoculated treatments. The soil $\mathrm{K}$ content was significantly higher in nine PSB treatments than other PSB treatments, $\mathrm{NP}_{0} \mathrm{~K}$, $\mathrm{NP}_{\mathrm{TCP}} \mathrm{K}$ and $\mathrm{NP}_{\mathrm{SSP}} \mathrm{K}$. The highest available $\mathrm{K}$ was recorded for $\mathrm{NP}_{\mathrm{TCP}} \mathrm{K}+P$ sp BIHB 756 . The available Ca was significantly higher in three PSB treatments than other PSB treatments, $\mathrm{NP}_{0} \mathrm{~K}, \mathrm{NP}_{\mathrm{TCP}} \mathrm{K}$ and $\mathrm{NP}_{\mathrm{SSP}} \mathrm{K}$.

\section{Discussion}

The organic acid production during solubilization of inorganic phosphates by the efficient phosphate-solubilizing strains of Pseudomonas trivialis, Pseudomonas poae, Pseudomonas fluorescens and Pseudomonas spp., corroborated their involvement in phosphate solubilization $[1,3,6]$.
Gluconic acid was the major organic acid produced as reported during phosphate solubilization by Pseudomonas sp. [16], P. fluorescens [17], Azospirillum spp. [18], Citrobacter sp. [19], and Pseudomonas corrugata [6]. The production of 2-ketogluconic, oxalic, malic, lactic, succinic, formic and citric acid in small quantities by Pseudomonas strains have also been reported during phosphate solubilization by Arthrobacter ureafaciens, Arthrobacter sp., Bacillus coagulans, B. megaterium, Chryseobacterium sp., Citrobacter koseri, Delftia sp., Enterobacter intermedium, Pseudomonas fluorescens, Rhodococcus erythropolis and Serratia marcescens $[3,6,16,20,21]$. None of Pseudomonas strains produced propionic acid unlike Bacillus megaterium strains during phosphate solubilization [3].

The results indicated that the quantity of organic acids produced differed with the nature of phosphate substrates and Pseudomonas strains (Tables 2, 3, 4, 5). The higher solubilization of TCP than URP, MRP and NCRP could possibly be due to the higher gluconic acid production in presence of TCP. The lower production of gluconic acid and lower TCP solubilization by Pseudomonas sp. BIHB 751 than other Pseudomonas strains substantiated the involvement of gluconic acid in solubilization of calciumbound phosphates. Succinic acid also appeared contribut- 
ing to TCP solubilization as it was produced by high TCPsolubilizing strains and not by low TCP-solubilizing Pseudomonas sp. BIHB 751 strain. The lack of oxalic acid production by efficient phosphate-solubilizing Pseudomonas strains signified non involvement of oxalic acid in TCP solubilization though this acid has been implicated besides citric, gluconic, lactic and succinic acids in phosphate solubilization in alkaline vertisols [20]. Pseudomonas sp. strain BIHB 751 producing the highest quantity of 2-ketogluconic acid but showing the lowest TCP and URP solubilization also differed from Enterobacter intermedium reported for the enhanced phosphate solubilization with increasing 2-ketogluconic acid production [21]. Likewise, no relationship could be ascertained between the quantity of organic acids produced and the solubilization of rock phosphates by Pseudomonas strains as the highest solubilization observed for NCRP among the rock phosphates was coupled to the lowest production of total organic acids (Tables 3, 4, 5). Previously also the quantities of solubilized phosphorus could not be correlated with the quantities of organic acids in the culture medium [22]. UPR, MRP and NCRP have fluorapatite structure with the highest substitution of phosphate with carbonate in NCRP [23]. The higher solubilization and lowered quantities of organic acids detected in the presence of NCRP could be due to the higher reactivity and greater diversion of organic acids in the neutralization of free carbonates in the solubilization of NCRP as compared to MRP and URP $[23,24]$. Likewise, the higher solubilization and higher production of organic acids in the presence of TCP could be attributed to its amorphous nature with simple structure and absence of any free carbonates as compared to the crystalline lattice structure of the rock phosphates [25].

Cluster analysis of organic acid profiles generated different groups revealing inter and intra-specific variation in the production of organic acids by Pseudomonas strains (Fig. 2). The strains clustered together and those standing outside the clusters or sub-clusters belonged to different Pseudomonas species characterized previously by $16 \mathrm{~S}$ rRNA gene sequencing $[8,9]$. The strains standing outside the clusters differed qualitatively and/or quantitatively from other strains in the production of organic acids (Tables 2, 3, 4, 5). The results implied that Pseudomonas strains are independent of their genetic relatedness in their phosphate-solubilizing ability and organic acid production even under similar set of culture conditions. Phosphate solubilization is a complex phenomenon which depends on the nutritional, physiological and growth conditions of the culture [26].

The enhanced growth and higher $\mathrm{N}, \mathrm{P}$ and $\mathrm{K}$ contents in maize with PSB treatments underlined the advantage of phosphate-solubilizing activity of microorganisms for plant growth promotion (Table 6 and 7). The increased growth and P uptake have been reported on PSB inoculations with Pseudomonas sp. and Serratia marcescens in maize [17], Pseudomonas fluorescens in peanut [27], Bacillus circulans in mungbean [28] and Pseudomonas sp. in wheat [29]. The TCP solubilization in soil by fluorescent Pseudomonas strains as evidenced by in vitro TCP solubilization, increased soil $\mathrm{P}$ availability and higher plant $\mathrm{P}$ content would be useful particularly in the cold deserts of Lahaul and Spiti where soil P deficiency is attributed mainly to the reaction of $\mathrm{P}$ with calcium carbonate and calcium sulphate forming insoluble di- and tricalcium phosphates. The rock phosphates recommended for acid soils are reportedly not effective in alkaline soils as $\mathrm{P}$ source for the crops [30]. The significantly higher plant growth and $\mathrm{N}, \mathrm{P}$, and $\mathrm{K}$ content in plant tissues and soil with some PSB treatments over $\mathrm{NP}_{\mathrm{SSP}} \mathrm{K}$ might be due to the immobilization of applied $P$ by native soil microbiota and physico-chemical reactions in the soil. The increased and continuous $P$ availability in the soil promotes biological nitrogen fixation [27]. No correlation among TCP solubilization, production of organic acids and plant growth promotion could be established as the highest solubilization and plant growth promoting activity was observed for $P$. trivialis BIHB 745 not showing the highest organic acid production. However, the lowest organic acid production and plant growth promotion by Pseudomonas sp. BIHB 751 showing the lowest TCP solubilization suggested that phosphate solubilization is an important mechanism of plant growth promotion. Pseudomonas strains exhibiting high TCP solubilization in vitro differed significantly in enhancing the plant growth in the soil indicating interplay of some other growth factors besides phosphate-solubilization (Tables 2, 6, and 7). Apart from making $\mathrm{P}$ available to the plants, phosphate-solubilizing microorganisms improve plant health directly by the production of phytohormones [31]. Pseudomonas strains have been reported to vary in their ability for phytohormone production [32-34]. The bacterial strains also differ in utilizing root exudates in producing biologically active substances and root colonizing ability known to influence the plant growth-promoting action of rhizobacteria [35]. Plant-microbe interaction is a complex phenomenon with the interplay of several mechanisms and environmental factors.

The decrease in soil pH in PSB treatments indicated the production of organic acids by Pseudomonas strains as also reported for phosphate-solubilizing Aspergillus niger and A. tubingensis [36]. However, less $\mathrm{pH}$ decline in soil during plant growth promotion experiments than phosphate solubilization in culture medium could be due to the buffering nature of soil [20]. The inorganic acids and $\mathrm{H}^{+}$ions of microbial origin and $\mathrm{H}^{+}$ions released from the plant roots during ammonium assimilation are also reported to influ- 
ence the soil $\mathrm{pH}[22,30,37]$. The studies have shown potential for plant growth promotion by $P$. trivialis BIHB 745, P. trivialis BIHB 747, Pseudomonas sp. BIHB 756 and $P$. poae $\mathrm{BIHB} 808$ in the presence of TCP as the phosphate source. The native phosphate-solubilizing and stress-tolerant Pseudomonas strains are expected to cohabitate as effective microbial inoculants with the crops grown in the cold deserts of Lahaul and Spiti.

\section{Conclusion}

The present study revealed that the innate ability of organic acid production by Pseudomonas strains is independent of their genetic relatedness. Significant difference in plant growth promotion among the efficient phosphate-solubilizing Pseudomonas strains point at the need for selecting the potential strains based on plant growth promotion in the soils supplemented with insoluble phosphates for their targeted application. The PSB strains with high potential for TCP solubilization appear promising for application in the Ca-rich and P-deficit soils in the cold deserts of Lahaul and Spiti for which field studies are required.

\section{Authors' contributions}

PV carried out the experiments on phosphate solubilization, organic acid profiling, plant growth promotion and chemical analyses, data analyses, and manuscript writing. AG contributed in experimental designing, interpretation of results, co-ordination and supervision of the experimental work, manuscript writing and editing.

\section{Acknowledgements}

Authors acknowledge the Director, Institute of Himalayan Bioresource Technology for providing the necessary facilities. The Council of Scientific and Industrial Research, Govt. of India, is also acknowledged for the financial support under the CSIR Network Project "Exploitation of India's Rich Microbial Wealth" (NWP 006). Thanks for the technical support are due to Mr. Ramdeen Prasad in chemical analyses and Mrs. Vijaylata Pathania for HPLC operation. The valuable suggestions by Dr. S. P. Sharma, Professor and Head and Dr. S.P. Dev, Scientist, Department of Soil Sciences, CSK HP KV (Agriculture University), Palampur (HP) are also acknowledged.

\section{References}

I. Goldstein $\mathrm{AH}$ : Recent progress in understanding the molecular genetics and biochemistry of calcium phosphate solubilization by Gram negative bacteria. Biology Agriculture and Horticulture 1995, I 2: |85-193.

2. Kim KY, Jordan D, McDonald GA: Enterobacter agglomerans, phosphate solubilizing bacteria and microbial activity in soil: effect of carbon sources. Soil Biology and Biochemistry 1998, 30:995-1003.

3. Chen YP, Rekha PD, Arun AB, Shen FT, Lai WA, Young CC: Phosphate solubilizing bacteria from subtropical soil and their tricalcium phosphate solubilizing abilities. Applied Soil Ecology 2006, 34:33-4I.

4. Whiting PH, Midgley M, Dawes EA: The regulation of transport of glucose, gluconate and 2-oxogluconate and of glucose catabolism in Pseudomonas aeruginosa. Biochemical Journal 1976, 154:659-668.

5. Rodriguez H, Fraga R: Phosphate solubilizing bacteria and their role in plant growth promotion. Biotechnology Advances 1999, 17:319-339.
6. Trivedi P, Sa T: Pseudomonas corrugata (NRRL B-30409) mutants increased phosphate solubilization, organic acid production, and plant growth at lower temperatures. Current Microbiology 2008, 56: | 140-I 44.

7. Botelho GR, Mendonça-Hagler LC: Fluorescent pseudomonads associated with the rhizosphere of crops- an overview. Brazilian Journal of Microbiology 2006, 37:40 I-4I6.

8. Gulati A, Rahi P, Vyas P: Characterization of phosphate-solubilizing fluorescent pseudomonads from the rhizosphere of seabuckthorn growing in the cold deserts of Himalayas. Current Microbiology 2008, 56:73-79.

9. Vyas P, Rahi P, Gulati A: Stress tolerance and genetic variability of phosphate-solubilizing fluorescent Pseudomonas from the cold deserts of the trans-Himalayas. Microbial Ecology 2009, 58:425-434.

10. Singh RP, Gupta MK: Soil and vegetation study of Lahaul and Spiti cold desert of western Himalayas. Indian Forester 1990, I 1 6:785-790.

II. Gulati A, Vyas P, Rahi P, Kasana RC: Plant growth promoting and rhizosphere competent Acinetobacter rhizosphaerae strain BIHB 723 from the cold deserts of Himalayas. Current Microbiology 2009, 58:37I-377.

12. McKeague JA: Manual on Soil Sampling and Methods of Analysis 2nd edition. Canadian Society of Soil Science, Ottawa, Canada; 1978.

13. Jackson ML: Soil Chemical Analysis Prentice Hall, New Delhi, India; 1973.

14. Olsen SR, Cole CV, Watanabe FS, Dean LA: Estimation of available phosphorus in soil by extraction with sodium bicarbonate. USDA Circ. 939. U.S. Government Printing Office, Washington, D.C; 1954.

15. Subbiah BV, Asija GL: A rapid procedure for the determination of available nitrogen in soils. Current Science 1956, 25:259-260.

16. Illmer P, Schiner F: Solubilization of inorganic phosphate by microorganisms isolated from forest soil. Soil Biology and Biochemistry 1992, 24:389-395.

17. Di Simine CD, Sayer JA, Gadd GM: Solubilization of zinc phosphate by a strain of Pseudomonas fluorescens isolated from a forest soil. Biology and Fertility of Soils 1998, 28:87-94.

18. Rodriguez H, Gonzalez T, Goire I, Bashan Y: Gluconic acid production and phosphate solubilization by the plant growth-promoting bacterium Azospirillum spp. Naturwissenschaften 2004, 91:552-555.

19. Patel DK, Archana G, Naresh Kumar G: Variation in the nature of organic acid secretion and mineral phosphate solubilization by Citrobacter sp. DHRSS in the presence of different sugars. Current Microbiology 2008, 56:168-174.

20. Gyaneshwar P, Naresh Kumar G, Parekh LJ: Effect of buffering on the phosphate solubilizing ability of microorganisms. World Journal of Microbiology and Biotechnology 1998, 14:669-673.

21. Hwangbo H, Park RD, Kim YW, Rim YS, Park KH, Kim TH, Suh JS Kim KY: 2-ketogluconic acid production and phosphate solubilization by Enterobacter intermedium. Current Microbiology 2003, 47:87-92.

22. Illmer P, Schinner F: Solubilization of inorganic calcium phosphates-solubilization mechanisms. Soil Biology and Biochemistry 1995, 27:257-263.

23. Narayanasamy G, Biswas DR: Phosphate rocks of India: Potentialities and constraints. Fertilizer News 1998, 43:21-28.

24. Bolland M: Effectiveness of rock phosphates. In Farm Note Department of Agriculture and Food, Government of Western Australia; 2007:2 I5

25. Kumari A, Kapoor KK, Kundu BS, Mehta RK: Identification of organic acids produced during rice straw decomposition and their role in rock phosphate solubilization. Plant Soil and Environment 2008, 54:72-77.

26. Reyes I, Bernier L, Simard PR, Antoun H: Effect of nitrogen source on the solubilization of different inorganic phosphates by an isolate of Penicillium rugulosum and two UV-induced mutants. FEMS Microbiology Ecology 1999, 28:28I-290.

27. Dey R, Pal KK, Bhatt DM, Chauhan SM: Growth promotion and yield enhancement of peanut (Arachis hypogaea L.) by application of plant growth promoting rhizobacteria. Microbiological Research 2004, I 59:37I-394.

28. Singh S, Kapoor KK: Effect of inoculation of phosphate-solubilizing microorganisms and arbuscular mycorrhizal fungus on 
mungbean grown under natural soil conditions. Mycorrhiza 1990, 7:249-253.

29. Babana $\mathrm{AH}$, Antoun $\mathrm{H}$ : Effect of Tilemsi phosphate rock-solubilizing microorganisms on phosphorus uptake and yield of field grown wheat (Triticum aestivum L.) in Mali. Plant and Soil 2006, 28:5।-58.

30. Gaur AC: Phosphate solubilizing microorganisms as biofertilizers. Omega Scientific Publishers, New Delhi; 1990.

31. Kloepper JW, Lifshitz R, Zablotowicz RM: Free living bacterial inocula for enhancing crop productivity. Trends in Biotechnology 1989, 7:39-44.

32. Ahmad F, Ahmad I, Khan MS: Indole acetic acid production by the indigenous isolates of Azotobacter and fluorescent Pseudomonas in the presence and absence of tryptophan. Turkish Journal of Biology 2005, 29:29-34.

33. Kang BR, Yang KY, Cho BH, Han TH, Kim IS, Lee MC, Anderson AJ, Kim YC: Production of indole-3-acetic acid in the plant-beneficial strain Pseudomonas chlororaphis $\mathrm{O6}$ is negatively regulated by the global sensor kinase GacS. Current Microbiology 2006, 52:473-476.

34. Tsavkelova EA, Cherdyntseva TA, Botina SG, Netrusov Al: Bacteria associated with orchid roots and microbial production of auxin. Microbiological Research 2007, 162:69-76.

35. Ladha JK, Triol AC, Ma LG, Darbey G, Caldo W, Ventura J, Watanabe $\mathrm{J}$ : Plant associated nitrogen fixation by five rice varieties and relationship with plant growth characteristics as affected by straw incorporation. Soil Science and Plant Nutrition 1986, 32:91-106.

36. Richa G, Khosla B, Sudhakara Reddy M: Improvement of maize plant growth by phosphate solubilizing fungi in rock phosphate amended soils. World Journal of Agricultural Sciences 2007, 3:48I-484

37. Flach EN, Quak W, Van Diest A: A comparison of the rock phosphate-mobilizing capacities of various crop species. Tropical agriculture 1987, 64:347-352.

Publish with Bio Med Central and every scientist can read your work free of charge

"BioMed Central will be the most significant development for disseminating the results of biomedical research in our lifetime. "

Sir Paul Nurse, Cancer Research UK

Your research papers will be:

- available free of charge to the entire biomedical community

- peer reviewed and published immediately upon acceptance

- cited in PubMed and archived on PubMed Central

- yours - you keep the copyright 